ARTICLE

DOI: $10.1038 / s 41467-018-06758-1$

\title{
High precision FRET studies reveal reversible transitions in nucleosomes between microseconds and minutes
}

\author{
Alexander Gansen', Suren Felekyan², Ralf Kühnemuth², Kathrin Lehmann (1) 1,2, Katalin Tóth', \\ Claus A. M. Seidel (i) ${ }^{2} \&$ Jörg Langowski id ${ }^{1}$
}

Nucleosomes play a dual role in compacting the genome and regulating the access to DNA. To unravel the underlying mechanism, we study fluorescently labeled mononucleosomes by multi-parameter FRET measurements and characterize their structural and dynamic heterogeneity upon $\mathrm{NaCl}$-induced destabilization. Species-selective fluorescence lifetime analysis and dynamic photon distribution analysis reveal intermediates during nucleosome opening and lead to a coherent structural and kinetic model. In dynamic octasomes and hexasomes the interface between the $\mathrm{H} 2 \mathrm{~A}-\mathrm{H} 2 \mathrm{~B}$ dimers and the $(\mathrm{H} 3-\mathrm{H} 4)_{2}$ tetramer opens asymmetrically by an angle of $\approx 20^{\circ}$ on a 50 and $15 \mu$ s time scale, respectively. This is followed by a slower stepwise release of the dimers coupled with DNA unwrapping. A mutation ( $\mathrm{H} 2 \mathrm{~A}$ $\mathrm{R} 81 \mathrm{~A}$ ) at the interface between $\mathrm{H} 2 \mathrm{~A}$ and $\mathrm{H} 3$ facilitates initial opening, confirming the central role of the dimer:tetramer interface for nucleosome stability. Partially opened states such as those described here might serve as convenient nucleation sites for DNA-recognizing proteins.

\footnotetext{
${ }^{1}$ DKFZ, Div. Biophysics of Macromolecules, 69120, Heidelberg, Germany. ${ }^{2}$ Heinrich-Heine-Universität, Lehrstuhl für Molekulare Physikalische Chemie, Düsseldorf 40225, Germany. These authors contributed equally: Alexander Gansen, Suren Felekyan, Ralf Kühnemuth, Kathrin Lehmann. Correspondence and requests for materials should be addressed to K.Tót. (email: kt@Dkfz-Heidelberg.de) or to C.A.M.S. (email: cseidel@hhu.de)
} 
T he eukaryotic genome is packaged into nucleosomes, in which about 150 base pairs of DNA are wrapped around a histone protein core $^{1,2}$. These are connected by short stretches (10-90 bp) of linker DNA to form a bead-on-a-string structure, which condenses into higher-order chromatin with the help of linker histones.

The nucleosome provides controlled access to DNA while keeping the compactness and integrity of the genetic material. Transient opening through thermal fluctuations can expose DNA binding $\operatorname{sites}^{3-5}$ even inside the nucleosome, where DNA unwrapping is much slower ${ }^{6}$. Other mechanisms, e.g. nucleosome repositioning or DNA replication ${ }^{7}$ may partially disrupt the nucleosome and help accessing buried DNA. The structure and dynamics of such transient intermediates are still poorly understood.

Single-molecule $(\mathrm{sm})$ techniques allow one to perform a detailed structural and kinetic analysis of transient structures in an ensemble under equilibrium conditions. Single-molecule Förster resonance energy transfer (smFRET) has been applied to studies of nucleosomes ${ }^{8-18}$ and nucleosome arrays with respect to structure and dynamics ${ }^{19,20}$. Combined with multi-parameter fluorescence detection (MFD), which classifies the fluorescence signal according to spectral response, lifetime and polarization, smFRET permits deep insight into the structural dynamics of biomolecules ${ }^{21-26}$, enabling detection of conformational substates during nucleosome opening ${ }^{12}$. Analyzing nucleosomes with different donor/acceptor positions by smFRET during saltinduced dissociation previously led us to postulate an intermediate open state with all histones still attached ${ }^{14,17}$, populated to about $1 \%$ under physiological conditions. Similar open structures were later confirmed by others ${ }^{27-29}$.

Recently a non-equilibrium stopped-flow ensemble study with small-angle X-ray scattering and ensemble FRET $^{30}$ analysis (time resolution $>5 \mathrm{~ms}$ ) examined the kinetics of nucleosome disassembly by diluting to very high salt concentrations (between 1.2 and $1.9 \mathrm{M} \mathrm{NaCl}$ ). The authors found evidence for unwrapped states with transition kinetics in the range of 50 milliseconds to seconds and suggested an ensemble of different extended DNA conformations formed by asymmetric and symmetric unwrapping with rather extended linker DNA arms where at least half the DNA was unwrapped from the histone core (see Fig. 4 in ref. ${ }^{30}$ ).

One could argue that the conditions, up to $>1 \mathrm{M} \mathrm{NaCl}$, are often outside the physiological range, but the detected intermediates may also be relevant for in vivo dissociation pathways. Here we use a direct approach with a network of four distinct FRET pairs and multi-parameter smFRET ${ }^{31}$ to unravel the very first opening steps of mononucleosomes reconstituted on the Widom 601 positioning sequence ("601 nucleosomes") ${ }^{32}$ under equilibrium conditions on very short time scales in the microsecond range. If the sample solution is stable and the measurement time is long enough, it has been shown by many groups that single-molecule techniques are perfectly suited to study the kinetics of exchange reactions in biomolecular systems ${ }^{33}$.

Based on the detailed insight into structural fluctuations, we derive a comprehensive kinetic scheme for nucleosome disassembly by systematically varying nucleosome concentration, ionic strength, label position and via introducing an $\mathrm{H} 2 \mathrm{~A}$ point mutation. We conclude that disassembly starts with breathing DNA and formation of dynamic "butterfly" octasomes ${ }^{14}$ opening on a $50 \mu$ s time scale by an angle of $\approx 20^{\circ}$ each that proceeds through weakening or disrupting of one or both interfaces between the H2A-H2B dimers and the $(\mathrm{H} 3-\mathrm{H} 4)_{2}$ tetramer. Eviction of one H2A-H2B dimer leads to a dynamic hexasome with opening dynamics on a $15 \mu$ s time scale which is followed by stepwise asymmetric DNA unwrapping and a slower release of the second dimer. While the loss of the first dimer occurs fast $(<300 \mathrm{~s}$ at $400 \mathrm{mM})$, the release of the second dimer is slow $(>10,000 \mathrm{~s})$. Our findings provide insight into the mechanism of nucleosome opening, leading to a kinetic scheme for nucleosome disassembly through at least seven species from initial fast unwrapping on the microsecond time scale up to the final dissociation during seconds or minutes.

\section{Results}

Nucleosome constructs. To analyze the dynamics of nucleosomes reconstituted on the non-palindromic Widom 601 sequence, we designed four differently labeled nucleosome constructs and one H2A mutant to study dimer release, asymmetric DNA opening and transient intermediates (Fig. 1 and Methods). To account for the asymmetry of the constructs, we denote the left side of the DNA sequence (forward (-) strand) by $\alpha$ and the other side $((+)$ strand) by $\beta)$. Analogously, we refer to the $(\mathrm{H} 2 \mathrm{~A}-\mathrm{H} 2 \mathrm{~B})$ dimer bound at the $\alpha$-side and $\beta$-side as Dimer $_{\alpha}$ and $\operatorname{Dimer}_{\beta}$, respectively.

Disassembly via asymmetric dimer loss and hexasome formation. Since nucleosome disassembly under force seems to be initiated by terminal DNA unwrapping followed by H2A-H2B dimer eviction ${ }^{34}$, we used the nucleosome construct H2B-Dy (Figs. 1, 2a) to analyze nucleosome disassembly with respect to the DNA ends. We followed salt-induced dimer loss measured by FRET between a donor on each H2A-H2B dimer and an acceptor on a DNA site near the dyad axis. We labeled the H2B with an efficiency of $\approx 45 \%$, so that we could study nucleosomes with one or two donors together with one or no acceptor. To compute the expected distances between the flexibly linked labels and the average FRET efficiency, we modeled dye position distributions (green and red clouds in Fig. 2a) by accessible volume (AV) simulations from the FRET-restrained positioning and screening (FPS) toolkit ${ }^{23,26}$. As the observed donor-acceptor (DA) distances are usually spatially averaged, the interdye distances recovered by fluorescence lifetime- or intensity-based FRET measurements differ slightly due to inherent different weighting. However, the knowledge of the AVs allows us to interconvert them (Methods and Supplementary Table 1). For convenience we present only the FRET-averaged DA distances, $\left\langle R_{\mathrm{DA}}\right\rangle_{\mathrm{E}}$. In the intact nucleosome $\left\langle R_{\mathrm{D}_{\alpha} \mathrm{A}}\right\rangle_{\mathrm{E}}$ for the labeled dimer at the $\alpha$-side is $\approx 60 \AA$ while $\left\langle R_{\mathrm{D}_{\beta} \mathrm{A}}\right\rangle_{\mathrm{E}}$ of the labeled dimer at the $\beta$-side is $\approx 79 \AA$ (Fig. 2a) and we could distinguish both dimers in the nucleosomes (Fig. 2b).

We used pulsed interleaved excitation (PIE) in a confocal microscope with multi-parameter fluorescence detection (MFD) 22 to analyze single diffusing nucleosomes (concentration $20 \mathrm{pM}$ labeled in presence of $980 \mathrm{pM}$ unlabeled nucleosomes) by their FRET properties and donor-to-acceptor dye stoichiometry $S^{35}$ values at different $\mathrm{NaCl}$ concentrations. In Fig. $2 \mathrm{~b}$ we show a representative two-dimensional burst frequency histogram of FRET efficiency, $E$, versus stoichiometry, $S$, for $150 \mathrm{mM} \mathrm{NaCl}$. Nucleosomes with a single donor, $\mathrm{D}_{\alpha} \mathrm{A}$ or $\mathrm{D}_{\beta} \mathrm{A}$, and with two donors $\mathrm{D}_{\alpha} \mathrm{D}_{\beta} \mathrm{A}$ were well resolved. With increasing $\mathrm{NaCl}$ concentration all three FRET-active species diminish in favor of the donor-only species (Fig. 2c). The double-donor $D_{\alpha} D_{\beta} A$ subspecies consists of intact nucleosomes which transit from the octasome into the hexasome by loss of one H2A-H2B dimer with $c_{1 / 2}=523 \pm 37 \mathrm{mM} \mathrm{NaCl}, c_{1 / 2}$ being the $\mathrm{NaCl}$ concentration at $50 \%$ dissociation as measure for nucleosome stability. The singledonor subspecies showed different stabilities against salt: $c_{1 / 2}$ $\left(\mathrm{D}_{\alpha} \mathrm{A}\right)=546 \pm 39 \mathrm{mM}$ and $c_{1 / 2}\left(\mathrm{D}_{\beta} \mathrm{A}\right)=866 \pm 33 \mathrm{mM}$, indicating that the $\mathrm{H} 2 \mathrm{~A}-\mathrm{H} 2 \mathrm{~B}$ dimer preferably evicts from the $\alpha$-side. This agrees with the asymmetric dissociation of the DNA (Fig. 3). There we compared salt-dependent DNA unwrapping of the two 


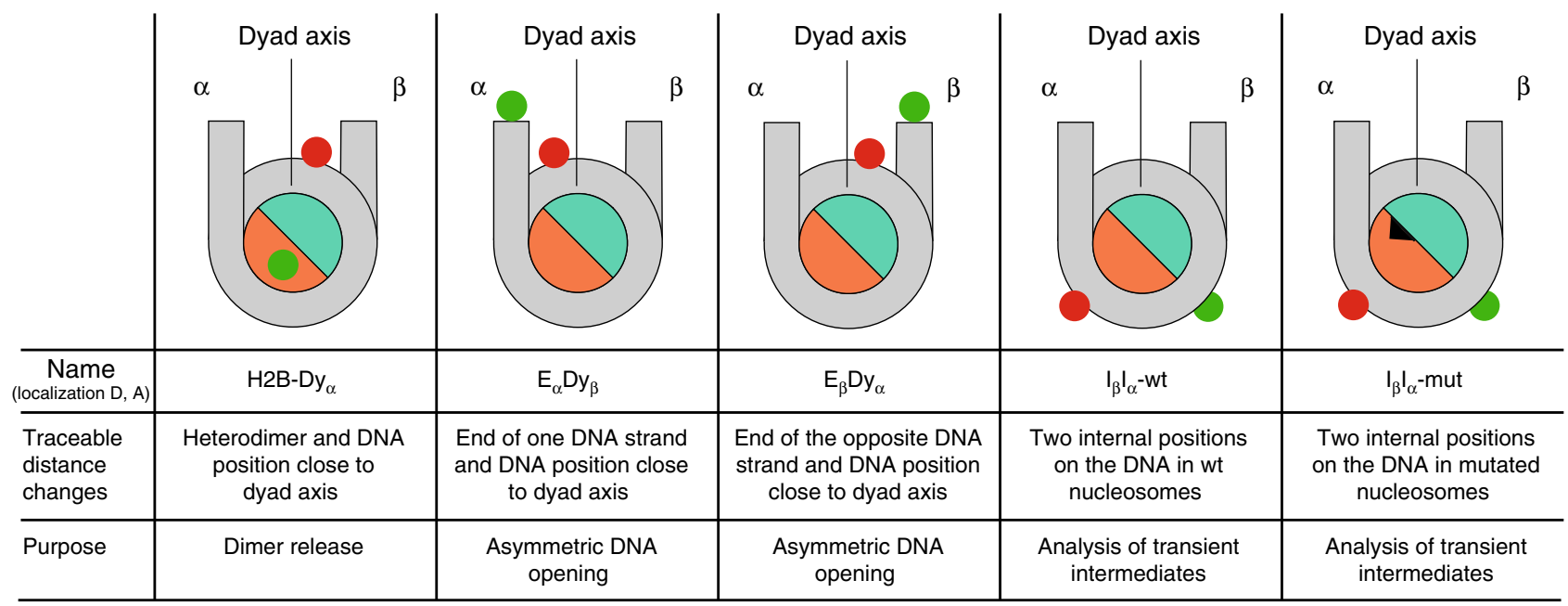

Fig. 1 Overview on FRET dye positions in the nucleosome constructs. The names of the constructs assign the position and side of the fluorescent labels: first the donor (green circle) and second the acceptor (red circle). In the cartoon representations, the $\mathrm{H} 2 \mathrm{~A}-\mathrm{H} 2 \mathrm{~B}$ dimers are shown in orange, the (H3-H4) tetramer in turquoise, DNA in gray. To account for the asymmetry, we denote by $\alpha$ the left side of the DNA sequence (forward (-) strand) with base pairs counted in negative numbers from the fragment center (position 0$)$. The other side is called $\beta$, with base pairs counted in positive numbers $((+)$ strand). The dye labeling positions on the DNA are given as relative base shifts to the middle of the sequence: Dy acceptor close to dyad axis at positions -15 and +15 , E donor labeling position at the end of the DNA strand at positions -85 and +85 , I internal labeling position on the DNA +41 (donor) and -53 (acceptor), $\mathrm{H} 2 \mathrm{~B}$ donor at position 112 of $\mathrm{H} 2 \mathrm{~B}$ using the $\mathrm{H} 2 \mathrm{~B}-\mathrm{T} 112 \mathrm{C}$ mutant, mut the H2A-R81A mutation is represented by a black triangle on the heterodimer

a

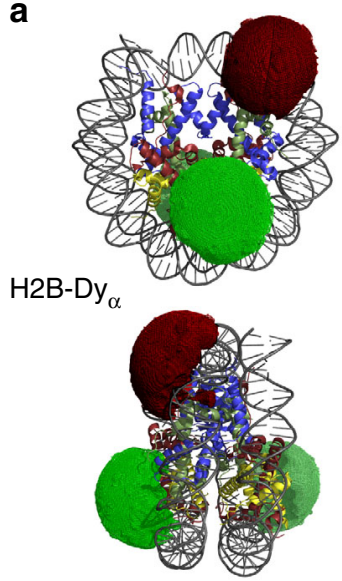

b

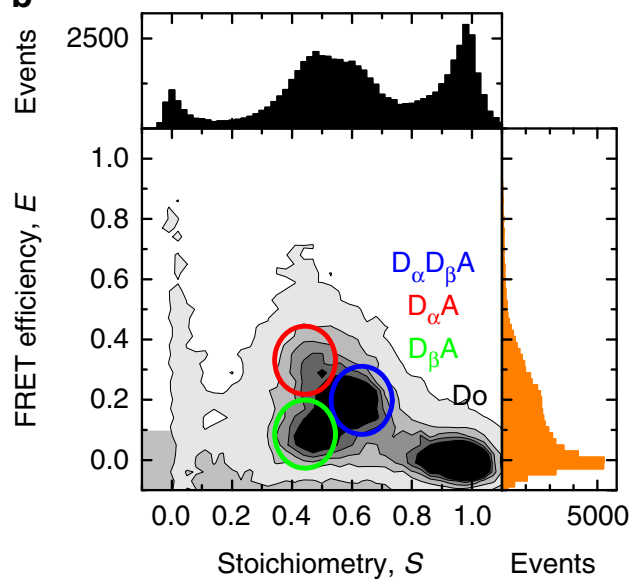

C

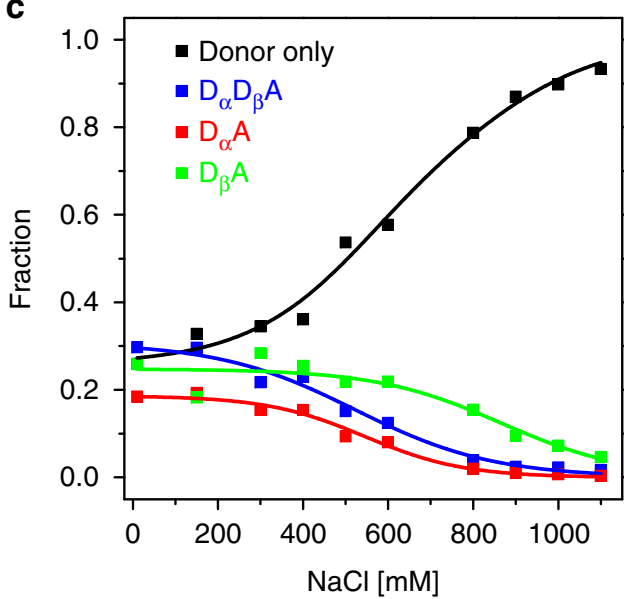

Fig. 2 MFD-PIE analysis of H2A-H2B dimer release. The nucleosome construct H2B-Dy $y_{\alpha}$ labeled with Alexa488 on each $\mathrm{H} 2 \mathrm{~A}-\mathrm{H} 2 \mathrm{~B}$ dimer and $\mathrm{Cy} 5$ on the DNA was used. a Crystal structure (3LZ1) with accessible fluorophore volumes for H2B-Dy ${ }_{\alpha}$ nucleosomes (green and red clouds for Alexa488 and Cy5, respectively) that describe the spatial distributions of the dyes. Histones are shown in blue (H3), pale green (H4), ruby (H2A), and yellow $(\mathrm{H} 2 \mathrm{~B})$. b FRET efficiency versus stoichiometry, $\mathrm{S}$, for $20 \mathrm{pM} \mathrm{H} 2 \mathrm{~B}-\mathrm{Dy}{ }_{\alpha}$ and $980 \mathrm{pM}$ unlabeled nucleosomes at $150 \mathrm{mM} \mathrm{NaCl}$. Subspecies are identified according to their position in the $2 \mathrm{D}$ distribution. c Relative population of donor-only, $\mathrm{D}_{\alpha} \mathrm{A}, \mathrm{D}_{\beta} \mathrm{A}$, and $\mathrm{D}_{\alpha} \mathrm{D}_{\beta} \mathrm{A}$ for $20 \mathrm{pM} H 2 \mathrm{~B}-\mathrm{Dy}$, and $980 \mathrm{pM}$ unlabeled nucleosomes as a function of $\mathrm{NaCl}$ concentration with fit to a sigmoid function (Supplementary Note 5, Supplementary Equation 36, global fit with $x_{\mathrm{Donor}}$ only $=1$

$\left.-x_{\mathrm{D} \alpha \mathrm{D} \beta \mathrm{A}}-x_{\mathrm{D} \alpha \mathrm{A}}-x_{\mathrm{D} \beta \mathrm{A}}\right) . \mathrm{D}_{\beta} \mathrm{A}$ nucleosomes prevail at significantly higher $\mathrm{NaCl}$ concentration than $\mathrm{D}_{\alpha} \mathrm{A}$ nucleosomes, indicating that $\mathrm{H} 2 \mathrm{~A}-\mathrm{H} 2 \mathrm{~B}$ heterodimers are predominantly evicted from the $\alpha$-side (see details in text)

nucleosome ends by multi-plate ensemble FRET measurements ${ }^{36}$ on constructs with double labeled DNA, $\mathrm{E}_{\alpha} \mathrm{Dy}_{\beta}$ and $\mathrm{E}_{\beta} \mathrm{Dy_{ \alpha }}$ (Supplementary Methods). Labeled nucleosomes were diluted to $600 \mathrm{pM}$ and incubated at different $\mathrm{NaCl}$ concentrations for 60 min. Both constructs behave similarly in the low- $\mathrm{NaCl}$ region $(<400 \mathrm{mM})$, where reversible DNA unwrapping at both ends is expected. We observe differences, though, at higher $\mathrm{NaCl}$ concentration where the loss of FRET indicates permanent detachment of the DNA ends from the histone core, likely accompanied by $\mathrm{H} 2 \mathrm{~A}-\mathrm{H} 2 \mathrm{~B}$ dimer eviction. The $\alpha$-side was less stably bound $\left(c_{1 / 2}\left(\mathrm{E}_{\alpha} \mathrm{Dy}_{\beta}\right)=631 \pm 6 \mathrm{mM}\right)$ than the $\beta$-side $\left(c_{1 / 2}\right.$
$\left.\left(\mathrm{E}_{\beta} \mathrm{Dy} \mathrm{y}_{\alpha}\right)=694 \pm 12 \mathrm{mM}\right)$. Thus, the $c_{1 / 2}$ values indicate that hexasome formation proceeds preferentially by dimer eviction from the $a$-side. Note that the $c_{1 / 2}$ values derived from species selective PIE-MFD and from ensemble measurements differ slightly because we probed different regions and species of the nucleosome (see below and Supplementary Note 5).

Dynamic intermediates are formed with altered DNA geometry. To analyze the kinetics of transient intermediates during disassembly we used internally labeled $\mathrm{I}_{\beta} \mathrm{I}_{\alpha}$-wt nucleosomes with 


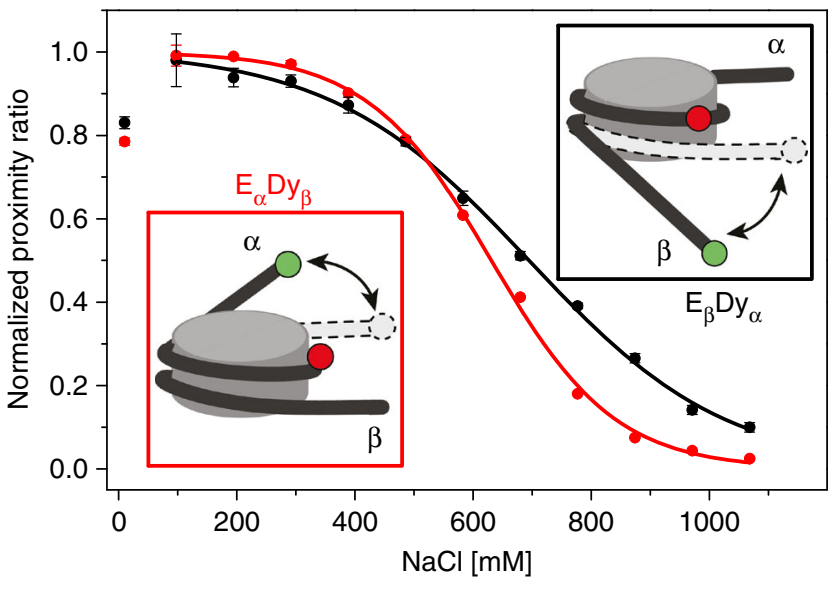

Fig. 3 Asymmetric unwrapping of DNA ends. Results of DNA unwrapping in the nucleosome constructs $E_{\alpha} D y_{\beta}$ and $E_{\beta} D y_{\alpha}$ (see Methods and Supplementary Note 5) studied by ensemble FRET studies. The dyes were placed at the DNA ends and near the dyad axis to report on unwrapping of each side through loss of FRET. For visualization, data were normalized to the maximal value of the proximity ratio at $100 \mathrm{mM} \mathrm{NaCl}$ and approximated by a sigmoidal function (Supplementary Note 5, Supplementary

Equation 36) to obtain the $c_{1 / 2}$ salt concentration. The $\beta$-side was significantly more resilient against salt-induced unwrapping than the $\alpha$-side. Error bars are standard errors from three replicates

both dyes attached to the dimer-binding region of DNA (Fig. 4a). For single-molecule MFD we first diluted the nucleosomes to a concentration of $20 \mathrm{pM}$ at $150 \mathrm{mM} \mathrm{NaCl}$ where intermediate structures appear at a physiological salt concentration ${ }^{12,17}$. Figure $4 \mathrm{~b}$ shows representative burst frequency populations for the burstwise analysis of intensity-derived FRET efficiency, $E$, and average fluorescence-weighted donor lifetime, $\left\langle\tau_{\mathrm{D}}\right\rangle_{\mathrm{F}}$, that allowed us to detect the presence of a fast dynamic exchange during the dwell time of a single particle in the detection volume ${ }^{37}$. The correlation between $E$ and $\left\langle\tau_{\mathrm{D}}\right\rangle_{\mathrm{F}}$ for molecules that remain in a single static conformation during detection is described by the black static FRET line (Supplementary Methods, Supplementary Equation 40). Any population that is shifted from this line to the right indicates that molecules fluctuate between multiple FRET species during the burst. The data reveal two quasi-static populations with the FRET species, LF (low FRET) and MF (mid FRET), that fall on the static FRET line with mean donor lifetimes of $\left\langle\tau_{\mathrm{D}}\right\rangle_{\mathrm{F}} \mathrm{LF} \approx 4.0 \mathrm{~ns}$ and $\left\langle\tau_{\mathrm{D}}\right\rangle_{\mathrm{F}} \mathrm{MF} \approx 2.9 \mathrm{~ns}$, respectively. A third population is slightly, but significantly, displaced from the static FRET line so that we refer to this broadened peak as the dynF population.

To resolve the composition of these populations with nanosecond time resolution, we accumulated subsets of selected single-molecule events belonging to a single population in Fig. 4b (highlighted in Supplementary Fig. 1a) and performed a global sub-ensemble analysis of their donor fluorescence decays (Supplementary Note 1, Supplementary Figs.1c-e). In agreement with the FRET-lines, we detected three global FRET species by their characteristic donor lifetimes and corresponding $\mathrm{DA}$ distances, $\left\langle R_{\mathrm{DA}}\right\rangle_{\mathrm{E}}$ : LF with $\left\langle\tau_{\mathrm{D}}\right\rangle_{\mathrm{F}}^{\mathrm{LF}} \approx 4.00 \mathrm{~ns}\left(\left\langle R_{\mathrm{DA}}\right\rangle_{\mathrm{E}} \approx 97 \AA\right)$, $\mathrm{MF}^{*}$ with $\left\langle\tau_{\mathrm{D}}\right\rangle_{\mathrm{F}} \mathrm{MF}^{*} \approx 2.65 \mathrm{~ns}\left(\left\langle R_{\mathrm{DA}}\right\rangle_{\mathrm{E}} \approx 57 \AA\right)$ and HF with $\left\langle\tau_{\mathrm{D}}\right\rangle_{\mathrm{F}} \mathrm{HF} \approx 1.30 \mathrm{~ns}\left(\left\langle R_{\mathrm{DA}}\right\rangle_{\mathrm{E}} \approx 46 \AA\right.$ ) (Supplementary Equation 1 , Supplementary Figs. 1c-e, Supplementary Tables 2-3). Considering the two quasi-static populations LF and MF, the corresponding FRET-species fractions recovered for LF (93\%) and MF (79\%), respectively, indicate that these populations are dominated by a single FRET species. The dynF population, however, consisted of a mixture of the FRET-species: $\mathrm{HF}$ and $\mathrm{MF}^{\star}$ that are in fast dynamic exchange. The exchange is well described by the dynamic FRET line connecting HF and $\mathrm{MF}^{*}$ (violet curve in Fig. 4b, Supplementary Methods, Supplementary Equation 41). The existence of this second transiently populated mid-FRET species $\left(\mathrm{MF}^{*}\right.$ ) which is in slow exchange with MF (yellow curve in Fig. 4b) is further justified at the end of this section. On the time scale of diffusion ( $3 \mathrm{~ms}$ ) we did not observe dynamic mixing between $\mathrm{MF}$ and $\mathrm{LF}$, suggesting that $\mathrm{MF} \rightleftarrows \mathrm{LF}$ transitions are significantly slower. Thus, LF can be considered static.

To extract absolute interdye distances and transition rate constants on the sub-millisecond time scale from the measured smFRET distributions, we characterized these subspecies further by dynamic photon distribution analysis (dynPDA) ${ }^{37}$ of the $\mathrm{D}$ and $\mathrm{A}$ fluorescence intensities ${ }^{38}$. DynPDA utilizes the stochastic nature of photon emission, background signal, spectral crosstalk, and dynamic exchange between states. We modeled the system with two exchanging states, $\mathrm{MF}^{*}$ and $\mathrm{HF}$, two static populations, MF and LF, and donor-only events (Fig. 4d). Since fluorescence lifetimes of $\mathrm{MF}$ and $\mathrm{MF}^{*}$ were similar, we approximated the DA distances of the MF and $\mathrm{MF}^{*}$ species by a joint Gaussian distance distribution (MF, $\mathrm{MF}^{*}$ ) with the FRET-averaged distance $\left\langle R_{\mathrm{DA}}{ }^{\mathrm{MF}}\right\rangle_{\mathrm{E}}$ and a half width $\sigma=2 \AA$ (Supplementary Fig. 2e). The average interdye distances from 4 independent measurements were $\left\langle R_{\mathrm{DA}} \mathrm{LF}\right\rangle_{\mathrm{E}}=(85.8 \pm 3.2) \AA,\left\langle R_{\mathrm{DA}}{ }^{\mathrm{MF}}\right\rangle_{\mathrm{E}}=(61.1 \pm 3.0) \AA$ and $\left\langle R_{\mathrm{DA}} \mathrm{HF}\right\rangle_{\mathrm{E}}=(46.1 \pm 0.8) \AA$, which agrees well with the value obtained by sub-ensemble fluorescence lifetime analysis of the dynF population (Supplementary Tables 2 and 3). At the concentration of $20 \mathrm{pM}$ nucleosome and $150 \mathrm{mM} \mathrm{NaCl}$, the forward and backward rates of the $\mathrm{MF}^{\star} \rightleftarrows \mathrm{HF}$ transition were $k_{\mathrm{MF}^{*} \rightarrow \mathrm{HF}}=(33.4 \pm 3.2) \mathrm{ms}^{-1}$ and $k_{\mathrm{HF} \rightarrow \mathrm{MF}^{*}}=(13.2 \pm 2.8) \mathrm{ms}^{-1}$ with a corresponding relaxation time $t_{\mathrm{R}}=(21.6 \pm 1.4) \mu \mathrm{s}$.

In summary, we assign the dominant species MF to the intact nucleosome (see Methods), since $\left\langle R_{\mathrm{DA}}{ }^{\mathrm{MF}}\right\rangle_{\mathrm{E}} \approx 61 \AA$ from dynPDA agrees very well with the distance estimated from the crystal structure 3LZ1 using the FPS toolkit $\left(\left\langle R_{\mathrm{DA}}\right\rangle_{\mathrm{E}}=60.3 \AA\right)$. LF is attributed to an open conformation, where the DNA is almost completely unbound. HF is considered as an intermediate species during disassembly with a lifetime in the range of tens of microseconds.

We have several corroborating arguments for the coexistence of MF and $\mathrm{MF}^{*}$ : (i) Within the precision of FRET the 2D MFD data of donor lifetime and intensity-based FRET efficiency indicate that the interdye distance of $\mathrm{MF}^{\star}$ is slightly shorter (by $\approx 4 \AA$ ) compared to MF (Fig. $4 \mathrm{~b}$ ). Because the distances are close to $R_{0}$, this corresponds to a significant change of FRET efficiency $\Delta E \approx 0.1$ that is readily detectable by sub-ensemble fluorescence decay analysis (Supplementary Figs. 1c-e). (ii) The dependence of the kinetic exchange rates on the overall nucleosome concentration, as described next, can only be explained by at least two species with very similar FRET properties: a dynamic $\left(\mathrm{MF}^{\star}\right)$ and a static (MF) subpopulation. (iii) Considering the disassembly pathway of mononucleosomes, mechanistic arguments postulate the existence of two structural states, octasome and hexasome, that have similar FRET efficiencies for the $\mathrm{I}_{\beta} \mathrm{I}_{\alpha}$ FRET-pair (see discussion).

Dissociation/association of $\mathrm{H} 2 \mathrm{~A}-\mathrm{H} 2 \mathrm{~B}$ dimers in intermediates. To reveal the nucleosome species involved in the $\mathrm{MF}^{*}-\mathrm{HF}$ transition, we investigated the dependence of this dynamic heterogeneity at $150 \mathrm{mM} \mathrm{NaCl}$ by varying the nucleosome concentration with unlabeled sample. DynPDA was employed to resolve the fractions and transition rate constants between the species. Typical MFD data at a total nucleosome concentration of $2 \mathrm{nM}$ are shown in Fig. 4c. The same three subspecies (LF, MF, dynF) were observed as at $20 \mathrm{pM}$ nucleosome concentration but with different 
a

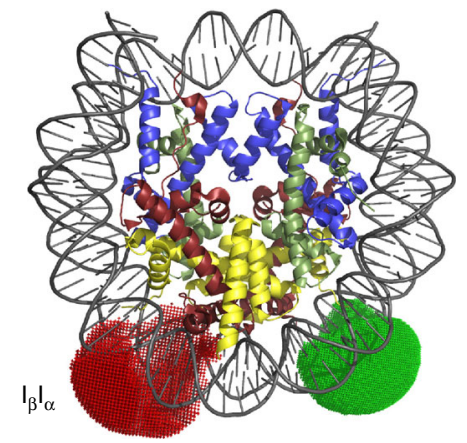

b

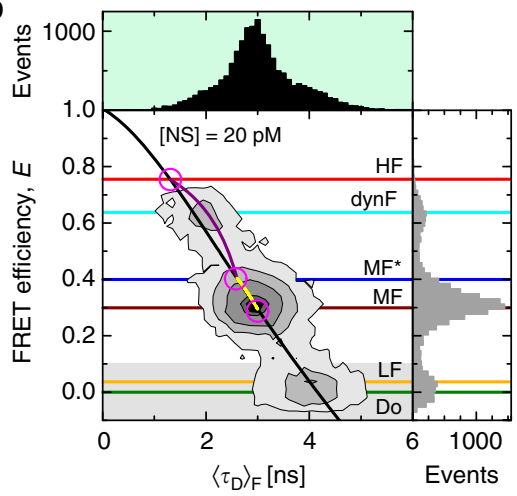

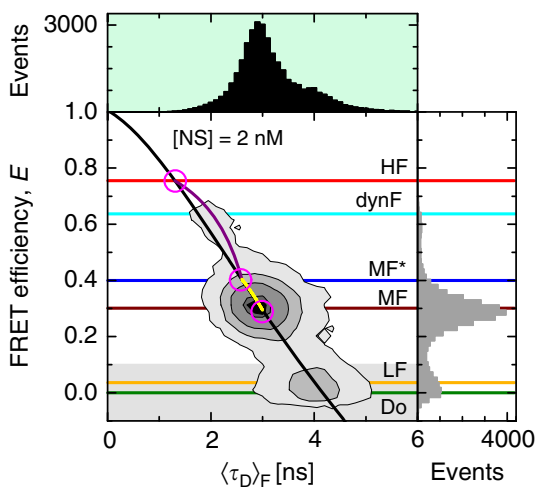

d

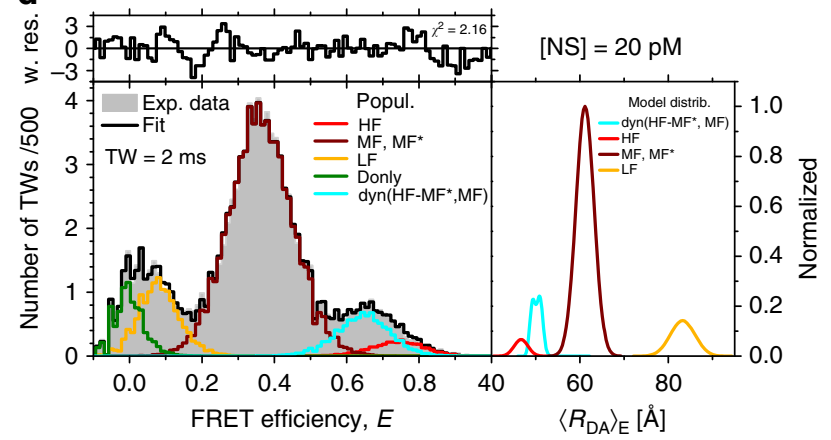

e

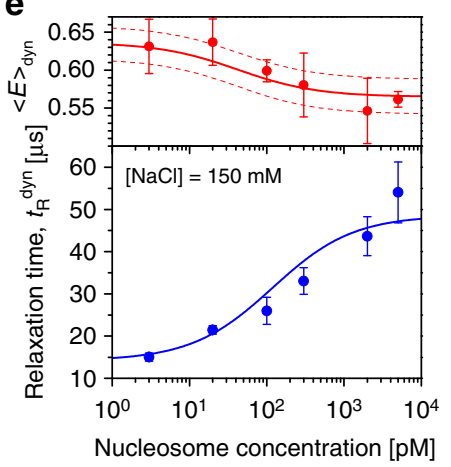

f Nucleosome concentration [pM]

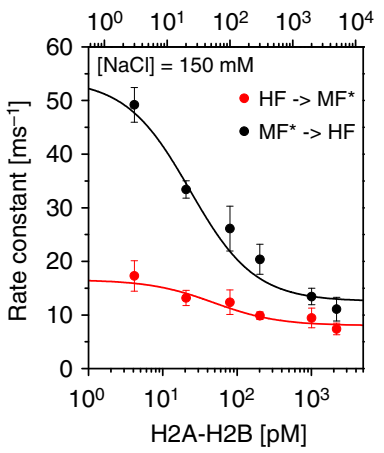

Fig. 4 Analysis of MFD smFRET data. Results of $\mathrm{I}_{\beta} \mathrm{l}_{\alpha}$-wt nucleosome construct studied at $150 \mathrm{mM} \mathrm{NaCl}$ labeled with Alexa488 (donor) and Alexa594 (acceptor). a Crystal structure 3LZ1 and accessible fluorophore volumes for donor (green) and acceptor (red). Histones are shown in blue (H3), pale green $(\mathrm{H} 4)$, ruby $(\mathrm{H} 2 \mathrm{~A})$ and yellow $(\mathrm{H} 2 \mathrm{~B})$. b, c FRET efficiency $(E)$ versus donor lifetime $\left(\left\langle\tau_{\mathrm{D}}\right\rangle_{\mathrm{F}}\right)$ for $20 \mathrm{pM}$ and $2 \mathrm{nM}$ (20 pM labeled and $1.98 \mathrm{nM}$ unlabeled) nucleosomes. Color coded horizontal lines correspond to FRET efficiency levels of different species. The $E-\left\langle\tau_{\mathrm{D}}\right\rangle_{\mathrm{F}}$ relations are presented by black line for static FRET species (Supplementary Methods, Supplementary Equation 40), violet line - for dynamic interconversion between $\mathrm{HF}\left(\left\langle\tau_{\mathrm{D}}\right\rangle_{\mathrm{F}}(\mathrm{HF})=1.3 \mathrm{~ns}\right)$ and $M^{\star}\left(\left\langle\tau_{D}\right\rangle_{F}\left(M F^{\star}\right)=2.65 \mathrm{~ns}\right)$ states and by yellow hypothetical dynamic FRET line connecting MF* and MF. $\mathbf{d}$ dynPDA of data shown in $\mathbf{b}$. Experimental FRET efficiency histogram for $2 \mathrm{~ms} \mathrm{TW}$ and fitted distributions for a model with two interconverting species (MF* and HF) and three static components as Gaussian-distributed distances (MF, LF, and donor-only). The quality of the fit is demonstrated by weighted residuals in the upper panel. The right panel shows the model with the intrinsic distribution of $\left\langle R_{\mathrm{DA}}\right\rangle_{\mathrm{E}}$ for the limit of large photon counts (shot noise free). e Relaxation times $t_{\mathrm{R}}^{\text {dyn }}=$

$\left(k_{\mathrm{MF}^{*} \rightarrow \mathrm{HF}}+k_{\mathrm{HF} \rightarrow \mathrm{MF}^{*}}\right)^{-1}$ and mean FRET efficiency $\langle E\rangle_{\mathrm{dyn}}=x_{\mathrm{MF}} E^{\mathrm{MF}}+x_{\mathrm{HF}} \mathrm{E}^{\mathrm{HF}}$ of dynamic species as a function of nucleosome concentration, calculated from dynPDA fits. $x_{\mathrm{MF}(\mathrm{HF})}$ were obtained from rate constants in $\mathbf{f}$ whereas $E^{\mathrm{MF}(\mathrm{HF})}$ were calculated from mean distances of interconverting states $\left(\left\langle R_{\mathrm{DA}}{ }^{\mathrm{MF}}\right\rangle_{\mathrm{E}}=\right.$ $61.2 \pm 1.5 \AA$ and $\left\langle R_{D A} H F\right\rangle_{E}=46.7 \pm 0.8 \AA$ ). $f$ Kinetic rate constants for the $M F^{\star} \rightleftarrows \mathrm{HF}$ transition as a function of the free $\mathrm{H} 2 \mathrm{~A}-\mathrm{H} 2 \mathrm{~B}$ concentration. Free $\mathrm{H} 2 \mathrm{~A}-$ $\mathrm{H} 2 \mathrm{~B}$ is estimated from the total nucleosome concentration and the different species fractions as obtained by PDA. The decrease of $k_{\mathrm{MF}^{\star} \rightarrow H F} \mathrm{With}$ increasing free heterodimer concentration implies the existence of two different $\mathrm{MF}^{\star}$ species. Error bars are standard errors from at least three different measurements. Lines are a weighted global fit of a four-state kinetic model to the data (see Supplementary Equations 16, 17)

proportions. At higher concentrations intact (MF) nucleosomes are stabilized at the expense of open (LF) and dynamic states (dynF). Interdye distances $\left\langle R_{\mathrm{DA}}{ }^{\mathrm{MF}}\right\rangle_{\mathrm{E}}$ and $\left\langle R_{\mathrm{DA}}{ }^{\mathrm{LF}}\right\rangle_{\mathrm{E}}$ were independent of sample concentration, indicating that the MF and LF structures remained unchanged (Supplementary Fig. 2a). Importantly, the average FRET efficiency $\langle E\rangle$ for the dynF peak decreased by 0.08 with increasing nucleosome concentration (Fig. 4e, top), while the relaxation time for the $\mathrm{MF}^{\star} \rightleftarrows \mathrm{HF}$ transition increased approximately by a factor of 3 (Fig. 4e, bottom).

This concentration dependence implies that the transition from $\mathrm{MF}^{\star}$ to $\mathrm{HF}$ involves an association/dissociation equilibrium, most likely the uptake/eviction of an $\mathrm{H} 2 \mathrm{~A}-\mathrm{H} 2 \mathrm{~B}$ dimer. Even if this process were diffusion limited, it would have relaxation times of $\mathrm{ms}$ or slower at sub-nanomolar concentrations. Thus, a single $\mathrm{MF}^{\star} \rightleftarrows \mathrm{HF}$ transition is not compatible with the observed fast microsecond kinetics. The simplest reaction scheme that accounts for all observations needs two dynF sub-populations (i.e. four nucleosome states). Each is characterized by a separate $\mathrm{MF}^{*}$-state in fast equilibrium with its HF counterpart: (i) an octasome, $\mathrm{O}$, that contains all core histones, with the equilibrium $\mathrm{O}_{\mathrm{cl}}^{\mathrm{MF} *} \rightleftarrows \mathrm{O}_{\mathrm{op}}^{\mathrm{HF}}$ and (ii) a hexasome, $\mathrm{H}$, that misses one $\mathrm{H} 2 \mathrm{~A}-\mathrm{H} 2 \mathrm{~B}$ dimer, with the equilibrium $\mathrm{H}_{\mathrm{cl}}^{\mathrm{MF} *} \rightleftarrows \mathrm{H}_{\mathrm{op}}^{\mathrm{HF}}$ (two dashed vertical boxes in the kinetic scheme later on). Dynamic states have the indices $\mathrm{cl}$ for the closed and op for the open conformation. Additionally, $\mathrm{O}_{\mathrm{st}}^{\mathrm{MF}}$ corresponds to the static octasome (PDB ID: 3LZ1) that is the majorly populated mid-FRET species MF. The $\mathrm{MF}^{*}$ species does not appear as a static subpopulation in the 2D MFD analysis, implying that they are in fast equilibrium with their HF counterparts and are not observable separately, but form a part of the dynF population. Based on the characteristic concentration dependence of the FRET efficiencies and anisotropy values (Supplementary Table 4) and recalling that the preferential dimer loss occurred at the $\alpha$-side (Fig. 2c), we hypothesize that the same FRET species, $\mathrm{MF}^{\star} \rightleftarrows \mathrm{HF}$, monitor fast parallel transitions within the dynamic octasome and the hexasome, respectively. The slow eviction/uptake of one H2A-H2B dimer that can occur at subnanomolar concentrations ${ }^{14}$ couples these two equilibria.

The states, $\mathrm{O}_{\mathrm{cl}}^{\mathrm{MF} *}, \mathrm{H}_{\mathrm{cl}}^{\mathrm{MF} *}$ and $\mathrm{O}_{\mathrm{st}}^{\mathrm{MF}}$, have very similar FRET efficiency values and are therefore difficult to distinguish by static FRET studies alone. However, the global analysis by dynPDA 
using this four state model (Supplementary Note 2) and the subensemble lifetime data suggest a simple reason for the concentration dependence of $k_{\mathrm{MF}^{*} \rightarrow \mathrm{HF}}$ in Fig. 4f: the forward rate $k_{\mathrm{MF}^{*} \rightarrow \mathrm{HF}}$ in the dynamic octasome population is slower than in the hexasome population. Since higher nucleosome concentrations decrease the hexasome population by pushing the majority of nucleosomes back via the dynamic octasome population into the intact, closed octasome species $\left(\mathrm{O}_{\mathrm{st}}^{\mathrm{MF}}\right), k_{\mathrm{MF} * \rightarrow \mathrm{HF}}$ drops. In contrast, the rate constant for the backward reaction $k_{\mathrm{HF} \rightarrow \mathrm{MF}^{*}}$ of the dynF population depends only moderately on [NS] concentration, since $\mathrm{O}_{\mathrm{op}}^{\mathrm{HF}}$ and $\mathrm{H}_{\mathrm{op}}^{\mathrm{HF}}$ have similar rates for the closing transition. In conclusion, we can distinguish these two transitions because they have distinct characteristic equilibrium constants (Fig. 4f, Supplementary Fig. 2b, c), so that the joint observables, the average FRET efficiency and the relaxation time, shift as function of the total nucleosome concentration (Fig. 4e).

The dimer:tetramer interface regulates the $\mathrm{MF}^{*}$ - HF transition. What is the driving force behind the $\mathrm{MF}^{\star} \rightleftarrows \mathrm{HF}$ transitions in nucleosomes? To answer this question, we used smFRET to analyze the stability of $20 \mathrm{pM} \mathrm{I}_{\beta} \mathrm{I}_{\alpha}$ nucleosome construct by varying $[\mathrm{NaCl}]$ from 5 to $1000 \mathrm{mM}$ (Fig. 5a). Below $600 \mathrm{mM}$ $[\mathrm{NaCl}], \mathrm{DNA}$ binding was intact for the majority of nucleosomes states. At higher salt, we observed a steep decrease for the MFand a correlated increase for LF-species fraction, suggesting gross DNA unwrapping, on a time scale of minutes to hours (Supplementary Fig. 3, Supplementary Table 6). Around 800-900 mM $[\mathrm{NaCl}]$ the majority of nucleosomes was found in a dynamic transition between $\mathrm{MF}^{*}$ and HF. The interdye distances did not vary significantly with salt, indicating that the structures of MF and LF remained unchanged (Supplementary Fig. 2d). Above $1 \mathrm{M}$ $\mathrm{NaCl}$, almost all nucleosomes were dissociated. The rate constants of the $\mathrm{MF}^{*} \rightleftarrows \mathrm{HF}$ transition changed with increasing salt concentration (Fig. 5b); $k_{\mathrm{MF}^{*} \rightarrow \mathrm{HF}}$ decreased while the back reaction rate constant increased (Fig. 5b), shifting the dynamic equilibrium between the two populations towards the closed configuration (Fig. 5c).

Previously, we suggested a disruption of the dimer:tetramer interface as an intermediate step in disassembly ${ }^{14}$. If this process was responsible for HF formation, mutations at the interface should affect $\mathrm{MF}^{\star} \rightleftarrows \mathrm{HF}$ dynamics. To test this hypothesis, we introduced the point mutation H2A-R81A in the dimer:tetramer interface at the position which is important for nucleosome stability 39,40 (Fig. 6a) and studied its impact on the dynF population. We first confirmed that the mutation did not affect nucleosome positioning and quality of reconstitution (Supplementary Fig. 4a), or fluorophore mobility as verified by MFD (Supplementary Fig. 4b, c). Salt-induced nucleosome disassembly in ensemble FRET (Fig. 6b) confirmed that mutated nucleosomes were significantly less stable than wt nucleosomes: $c_{1 / 2}\left(\mathrm{I}_{\beta} \mathrm{I}_{\alpha}-\mathrm{wt}\right)=$ $783 \pm 5 \mathrm{mM}, c_{1 / 2}\left(\mathrm{I}_{\beta} \mathrm{I}_{\alpha}\right.$-mut $)=618 \pm 7 \mathrm{mM}$ (standard errors, $N=$ 6). Mutated nucleosomes were also more sensitive to low [NS] concentration $(20 \mathrm{pM})$ so that we used $100 \mathrm{pM}$ total nucleosome concentration for the smFRET measurements to map the structural heterogeneities of $\mathrm{H} 2 \mathrm{~A}-\mathrm{R} 81 \mathrm{~A}$ and wt nucleosomes between 150 and $1000 \mathrm{mM} \mathrm{NaCl}$. While both constructs behaved similarly at $150 \mathrm{mM}$ salt (Supplementary Fig. 4d, e), even moderately higher $\mathrm{NaCl}$ concentrations revealed considerable differences (Fig. 6c, d). H2A-R81A mutation promoted conversion of the MF species into dynF without increasing the LF species fraction on the time scale of $1000 \mathrm{~s}$. Gross dissociation and an increase in LF occurred only above $600 \mathrm{mM} \mathrm{NaCl}$ (Supplementary Fig. $4 \mathrm{f}-\mathrm{i})$. Finally, kinetic rates for the $\mathrm{MF}^{\star} \rightleftarrows \mathrm{HF}$ transition were determined by dynPDA. Again, the H2A-R81A mutation did not alter kinetic rates at $150 \mathrm{mM} \mathrm{NaCl}$, but caused significantly increased kinetic rates at intermediate ionic strength with a doubled opening rate constant $k_{\mathrm{MF}^{*} \rightarrow \mathrm{HF}}$ (Supplementary Fig. 4j, k).

Taken together, the data on $\mathrm{I}_{\beta} \mathrm{I}_{\alpha}$-mut nucleosomes support our hypothesis that partial opening of the dimer:tetramer interface is responsible for the $\mathrm{MF}^{\star} \rightleftarrows \mathrm{HF}$ transitions. Its dependence on nucleosome concentration (Fig. 4f) indicates that the histone core contains at least one dimer in both dynF states.

Geometric structural models of the $\mathrm{HF}$ and $\mathrm{MF}^{\star}$ species. Finally, we tried to connect the kinetic scheme with the FRET species, $\mathrm{MF}^{*}$ and $\mathrm{HF}$, with structural candidates for subnucleosomal particles that can open up at the dimer:tetramer interface by an angle $\theta$ using a simplified geometric model of the nucleosome (Supplementary Note 3). The model was
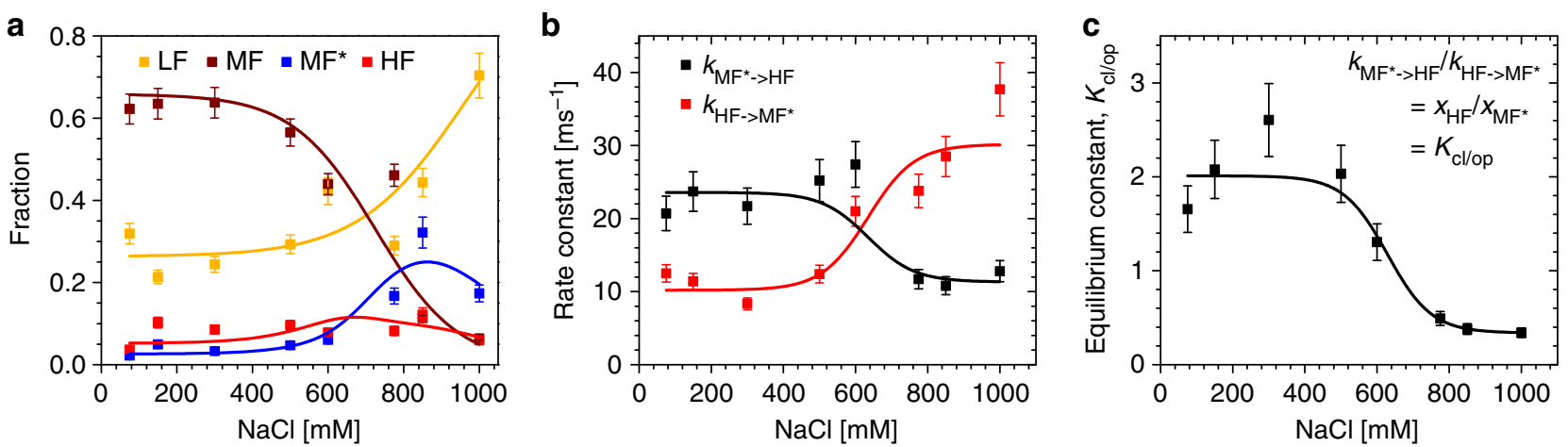

Fig. 5 Kinetic parameters of $\mathrm{I}_{\beta} \mathrm{I}_{\alpha}$-wt nucleosomes as function of $\mathrm{NaCl}$ concentration. The dependence of fractions of the four FRET species on the NaCl concentration were determined from single molecule measurements of $20 \mathrm{pM} \mathrm{I}_{\beta} \mathrm{I}_{\alpha}$-wt nucleosomes by dynPDA. The transitions are described by weighted fits to a sigmoidal function (Supplementary Note 5, Supplementary Equation 36). a Salt-dependence of the static fractions $x_{i}$ of $i=\mathrm{LF}, \mathrm{MF}, \mathrm{MF}{ }^{\star}$, and HF. Intact nucleosomes (MF) are dominant at low salt concentrations but decrease rapidly above $600 \mathrm{mM} \mathrm{NaCl}$ in favor of $\mathrm{LF}$ and $\mathrm{MF}^{\star}$. The fraction of nucleosomes that was found in a dynamic transition between $\mathrm{MF}^{\star}$ and $\mathrm{HF}$ peaks around $800-900 \mathrm{mM} \mathrm{NaCl}$. Global fit with $x_{\mathrm{MF}}=\left(1-x_{\mathrm{LF}}-x_{\mathrm{MF}}\right) /\left(1+K_{\mathrm{cl}}\right.$ $\left.{ }_{\mathrm{op}}\right)$ and $x_{\mathrm{HF}}=\left(1-x_{\mathrm{LF}}-x_{\mathrm{MF}}\right)^{\star} K_{\mathrm{cl} / \mathrm{op}} /\left(1+K_{\mathrm{cl} / \mathrm{op}}\right)$. The salt-dependent equilibrium constant $K_{\mathrm{cl} / \mathrm{op}}$ was obtained from the fit in c. $c_{1 / 2}(\mathrm{MF})=725 \pm 25 \mathrm{mM}, \mathrm{c}_{1 / 2}$ $(\mathrm{LF})=957 \pm 41 \mathrm{mM}$. b Salt-dependent kinetic rates for the $\mathrm{MF}^{\star} \rightleftarrows \mathrm{HF}$ transition. Transitions from $\mathrm{MF}^{\star}$ to $\mathrm{HF}$ are suppressed at higher $\mathrm{NaCl}$ concentrations, while the back reaction from $\mathrm{HF}$ to $\mathrm{MF}^{\star}$ is promoted by increasing salt. Global fit with $c_{1 / 2}=635 \pm 60 \mathrm{mM}$. c Equilibrium constant $K_{\mathrm{cl} / \mathrm{op}}$ as obtained from the rates in $\mathbf{b}: K_{\mathrm{cl} / \mathrm{op}}=k_{\mathrm{MF}^{*} \rightarrow \mathrm{HF}} / k_{\mathrm{HF} \rightarrow \mathrm{MF}^{*}}=x_{\mathrm{HF}} / x_{\mathrm{MF}^{*}} . c_{1 / 2}\left(\mathrm{~K}_{\mathrm{cl} / \mathrm{op}}\right)=629 \pm 34 \mathrm{mM}$. Error bars are standard errors from at least three independent measurements 
a

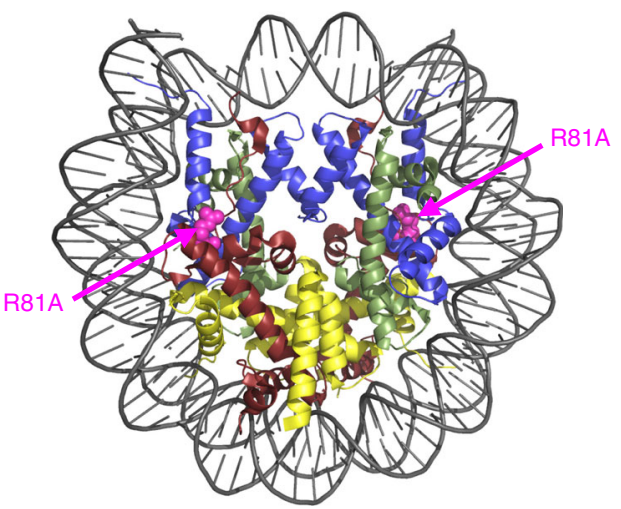

c

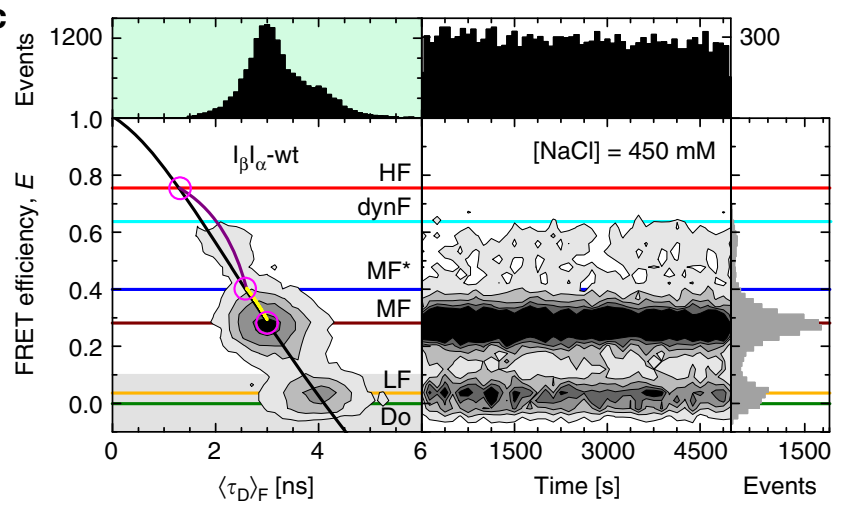

b

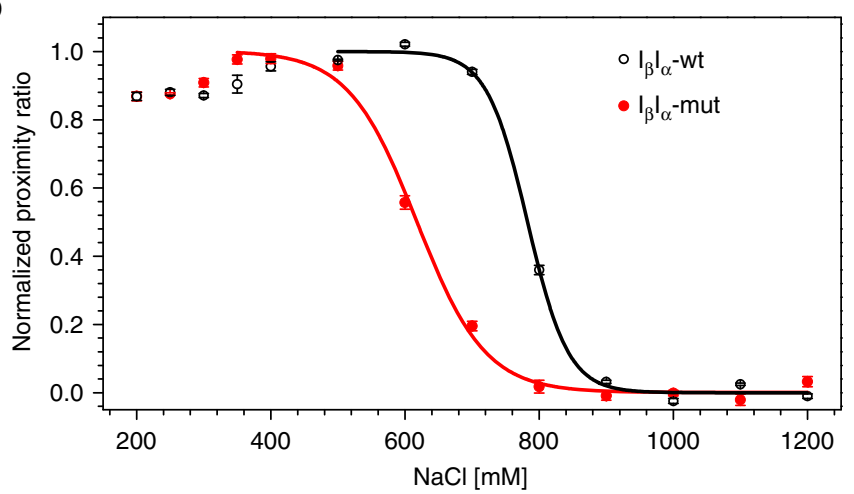

d

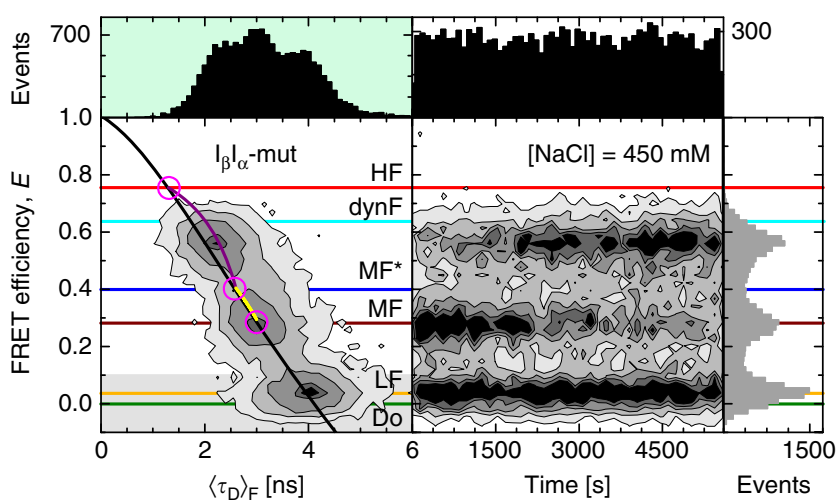

Fig. 6 Effect of the point mutation R81A in H2A on nucleosome dynamics and disassembly. a Crystal structure (3LZ1) with the H2A-R81A mutation (shown in a space filling magenta representation) that modifies the charge at the dimer:tetramer interface. Histones are shown in blue (H3), pale green (H4), ruby $(\mathrm{H} 2 \mathrm{~A})$ and yellow $(\mathrm{H} 2 \mathrm{~B})$. b Ensemble FRET analysis of salt-induced disassembly of $\mathrm{I}_{\beta} \mathrm{l}_{\alpha}$-wt and $\mathrm{I}_{\beta} \mathrm{I}_{\alpha}$-mut nucleosomes. Nucleosomes carrying mutated $\mathrm{H} 2 \mathrm{~A}$ are more susceptible to elevated ionic strength than their non-mutated counterpart; $c_{1 / 2}\left(I_{\beta} I_{\alpha}\right.$-mut $)$ is about $165 \mathrm{mM}$ lower than $c_{1 / 2}\left(\mathrm{I}_{\beta} \mathrm{I}_{\alpha}\right.$-wt). Average values from 6 independent measurements were: $c_{1 / 2}\left(\mathrm{I}_{\beta} \mathrm{l}_{\alpha}-\mathrm{wt}\right)=783 \pm 5 \mathrm{mM}$ and $c_{1 / 2}\left(\mathrm{I}_{\beta} \mathrm{l}_{\alpha}\right.$-mut $)=618 \pm 7 \mathrm{mM}$. c MFD-smFRET analysis of $100 \mathrm{pM} \mathrm{I}_{\beta} \mathrm{l}_{\alpha}$-wt nucleosomes at $450 \mathrm{mM} \mathrm{NaCl}$. The majority of nucleosomes are found in MF; all three subspecies (LF, MF and dynF) remained stable over time. $\mathbf{d}$ MFDsmFRET analysis of $100 \mathrm{pM} \mathrm{I} \mathrm{I}_{\alpha}$-mut nucleosomes at $450 \mathrm{mM} \mathrm{NaCl}$. Over time, a significant portion of intact nucleosomes (MF) transit into the dynamic FRET state. The fraction of fully open nucleosomes (LF), however, did not change over time. All colored lines in panels $\mathbf{c}$ and $\mathbf{d}$ match the lines in Fig. $4 \mathrm{a}$, $\mathrm{C}$. Error bars are standard errors from three replicates

parameterized based on the crystal structure (PDB ID: 3LZ1) and the simulation of accessible fluorophore space in $\mathrm{I}_{\beta} \mathrm{I}_{\alpha}$ nucleosomes to determine distances between the mean positions (mp) of donor and acceptor, $R_{\mathrm{mp}}{ }^{26}$ (Supplementary Note 3 ), which were subsequently converted to the experimentally observable $\left\langle R_{\mathrm{DA}}\right\rangle_{\mathrm{E}}$. The computed distance for the intact nucleosome is $\left\langle R_{\mathrm{DA}}\right\rangle_{\mathrm{E}}=61$ $\AA$, in excellent agreement with our experimental data. Distances in hexasomes were computed by removing one of the dimers and allowing DNA to exit tangentially from the disrupted dimer:tetramer interface (Fig. 7). This can be justified since the distance between tetramer end and dye on the free DNA segment is less than $20 \mathrm{bp}$, much smaller than its persistence length. Likewise, the z-pitch of the free DNA segment was assumed unchanged. For hexasomes missing the dimer at the $\beta$-side we computed $\left\langle R_{\mathrm{DA}} \beta\right\rangle_{\mathrm{E}}$ $=56 \AA$, while dimer removal at the acceptor site ( $\alpha$-side) yielded $\left\langle R_{\mathrm{DA}}{ }^{\alpha}\right\rangle_{\mathrm{E}}=58 \AA$. Recall that the latter is the dominant hexasome species in $\mathrm{MF}^{*}$ due to asymmetric dimer eviction. The small difference in distance between the intact nucleosome and the hexasome species agrees with the slightly smaller donor fluorescence lifetime measured for $\mathrm{MF}^{*}$ in our MFD experiments.

For the modelling we considered (i) single opening of the nucleosome at one of the dimers (wine and orange lines), (ii) opening of the octasome at both dimer interfaces (light blue line), and (iii) opening of a hexasome that is already partially open (gray and black lines). The computed interdye distances for all potentially dynamic species as a function of $\theta$ are shown in Fig. 7 together with the average experimental distances for both transient FRET species $\mathrm{HF}$ and $\mathrm{MF}^{*}$ (red and blue ribbons). In the intact nucleosome $\left\langle R_{\mathrm{DA}}\right\rangle_{\mathrm{E}}$ depends only weakly on $\theta$ (wine and orange curves) and crosses the $\mathrm{MF}^{*}$ values at about $15-20^{\circ}$. If only one histone dimer detaches, one would need $\theta>70^{\circ}$ to reach the HF interdye distance. Such a large angle would require sharp overbending of DNA, which is energetically unfavorable. However, a simultaneous opening of both interfaces in octasomes (light blue curve) or in hexasomes (black and gray curves) by $\approx 20^{\circ}$ would already suffice to produce a mean dye distance comparable to $\left\langle R_{\mathrm{DA}}{ }^{\mathrm{HF}}\right\rangle_{\mathrm{E}}$ (Fig. 7). This corroborates our idea, that $\mathrm{HF}$ might be an open octasome $\mathrm{O}_{\mathrm{op}}^{\mathrm{HF}}$ or hexasome $\mathrm{H}_{\mathrm{op}}^{\mathrm{HF}}$ state and $\mathrm{MF}^{\star}$ is a closed octasome $\mathrm{O}_{\mathrm{cl}}^{\mathrm{MF} *}$ or hexasome $\mathrm{H}_{\mathrm{cl}}^{\mathrm{MF} *}$ state.

Furthermore, we need to rule out other mechanisms that could result in similar FRET changes. Extended DNA breathing for example would not affect the mean dye distance until a significant portion of DNA is unpeeled. This would increase donor mobility, which we did not observe in our anisotropy studies (Supplementary Table 4 and Supplementary Note 6). Nucleosome gaping - an out-of-plane motion of the two DNA turns - would increase the mean dye distance, contrary to the decrease observed in HF. Moreover, the characteristic time scale for this process is a few minutes ${ }^{29}$, orders of magnitude slower than the $\mathrm{MF}^{*} \rightleftarrows \mathrm{HF}$ transition. 

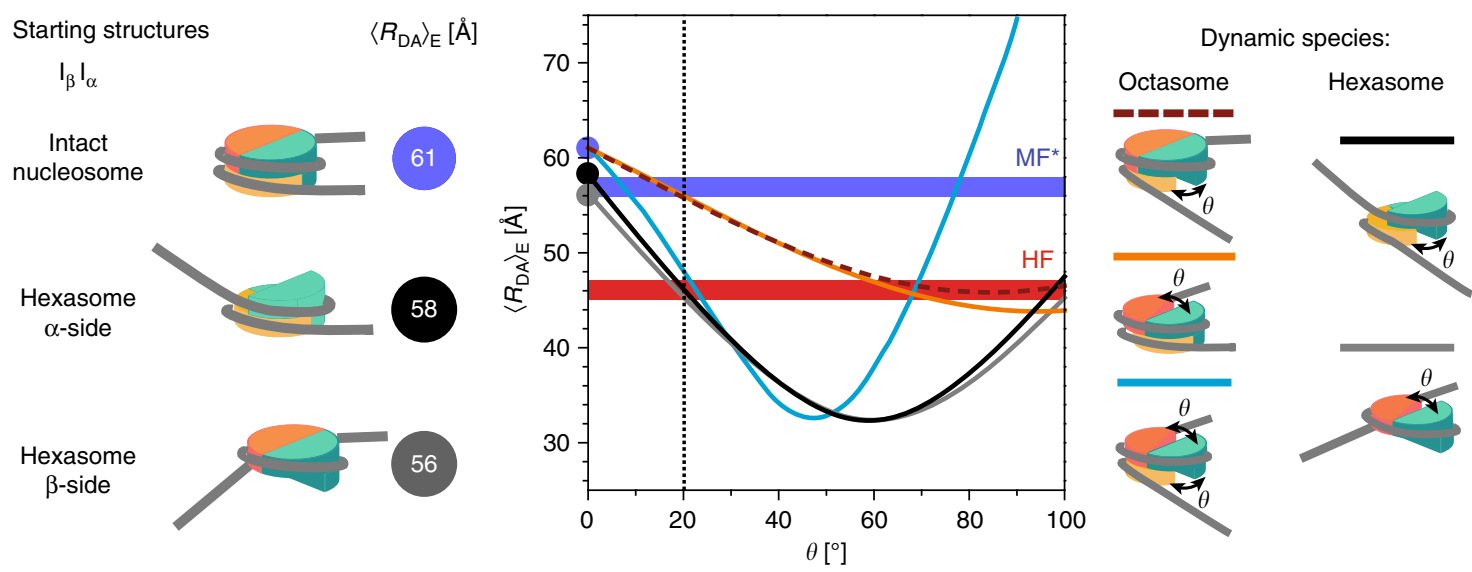

Fig. 7 A geometric model for nucleosome dynamics. All details on the parameterization of the intact nucleosome and definitions of relevant parameters and the computer code are given in Supplementary Note 3. The $(\mathrm{H} 3-\mathrm{H} 4)_{2}$ tetramer is shown in turquoise, while $\mathrm{H} 2 \mathrm{~A}-\mathrm{H} 2 \mathrm{~B}$ heterodimers are shown in orange $\left(\alpha\right.$-side) and dark yellow ( $\beta$-side). The interdye distances $\left\langle R_{\mathrm{DA}} \mathrm{MF}^{\star}\right\rangle_{\mathrm{E}}$ are computed for the following three starting structures (opening angle $\theta=0^{\circ}$ ): the intact nucleosome and both possible hexasomes with an open $\alpha$-side and $\beta$-side, respectively. We compute the geometry of potentially dynamic nucleosome species as a function of the opening angle $\theta$ (between the $\mathrm{H} 2 \mathrm{~A}-\mathrm{H} 2 \mathrm{~B}$ dimer and the $(\mathrm{H} 3-\mathrm{H} 4)_{2}$ tetramer) for five scenarios dimer:tetramer opening: (i, ii) intact nucleosomes (octasomes), where opening occurs either only on the $\alpha$-side (orange line) or only on the $\beta$-side (wine dashed line), (iii) intact nucleosomes (octasomes) with simultaneous opening on both sides with equal angles (cyan line) and (iv, $v$ ) hexasomes that are missing the dimer at the $\alpha$-side (black line) or at the $\beta$-side (gray line) with additional opening on the other so far closed side. The blue and red ribbons represent the ranges of experimentally determined average interdye distances for $\mathrm{MF}^{\star}\left(\left\langle R_{\mathrm{DA}} \mathrm{MF}^{\star}\right\rangle_{\mathrm{E}}=(57 \pm 1) \AA\right)$ and $\mathrm{HF}\left(\left\langle R_{\mathrm{DA}} \mathrm{HF}\right\rangle_{\mathrm{E}}=(46 \pm 1) \AA\right)$, respectively. Our model predicts an simultaneous and equal opening by $\approx 20^{\circ}$ within a hexasome or an octasome, while rather unrealistic angles greater than $70^{\circ}$ would be required for nucleosome species with only one open dimer:tetramer interface

Intermediates of nucleosome disassembly. Considering the stepwise nucleosome destabilization with increasing $\mathrm{NaCl}$ concentration, our results obtained by four different label positions (summarized in Supplementary Table 5) and the H2A-R81A mutant (Fig. 1) led to a coherent picture of the structural features of the corresponding nucleosome states. In the dynamic octasome the DNA was not significantly unwrapped and the first saltinduced event was the dimer release on the $\alpha$-side $\left(c_{1 / 2}(\mathrm{H} 2 \mathrm{~B}\right.$ $\left.\mathrm{Dy}_{\alpha}\left(\mathrm{D}_{\alpha} \mathrm{A}\right)\right)=546 \pm 39 \mathrm{mM}$, Fig. $2 \mathrm{c}$ ) that resulted in the formation of dynamic hexasomes as the majorly populated nucleosome state. Further $\mathrm{NaCl}$ increase led to a stepwise unwrapping of the DNA ends: firstly, on the $\alpha$-side $\left(c_{1 / 2}\left(\mathrm{E}_{\alpha} \mathrm{Dy}_{\beta}\right)=631 \pm 6 \mathrm{mM}\right.$, Fig. 3) with a simultaneous change of the equilibrium constant, $K_{\mathrm{cl} / \mathrm{op}}$, for the exchange between closed and open nucleosome species (cl/op) favoring the more stable closed state $\mathrm{H}_{\mathrm{cl}}^{\mathrm{MF} *}\left(c_{1 /}\right.$ ${ }_{2}\left(K_{\mathrm{cl} / \mathrm{op}}\right)=629 \pm 34 \mathrm{mM}$, Fig. $\left.5 \mathrm{c}\right)$; secondly, on the $\beta$-side $\left(c_{1 /}\right.$ ${ }_{2}\left(\mathrm{E}_{\beta} \mathrm{Dy} \mathrm{y}_{\alpha}\right)=694 \pm 12 \mathrm{mM}$, Fig. 3). Next, DNA opened up also within the hexasome $\left(\left(c_{1 / 2}\left(\mathrm{I}_{\beta} \mathrm{I}_{\alpha}\right)=783 \pm 5 \mathrm{mM}\right.\right.$, Fig. $\left.6 \mathrm{~b}\right)$ and the tetrasome with extended DNA was formed by release of the second dimer $\left(c_{1 / 2}\left(\mathrm{H} 2 \mathrm{~B}-\mathrm{Dy}_{\alpha}\left(\mathrm{D}_{\beta} \mathrm{A}\right)\right)=866 \pm 33 \mathrm{mM}\right.$, Fig. 2). Notably, most salt-induced nucleosome transitions have broad half-widths $b$ in the range of 100-150 mM, (Supplementary Table 5) which indicates heterogeneity with multiple intermediates.

Combining the dynamic and structural features of the nucleosome states revealed by our smFRET studies, we suggest a disassembly pathway of the intact nucleosome $\mathrm{O}_{\mathrm{st}}^{\mathrm{MF}}$ with at least five major steps and seven characteristic intermediates (Fig. 8). Moreover, we determined all rate and equilibrium constants by detailed kinetic analysis of the MFD data (relaxation times and dynPDA species fractions, see Supplementary Note 2) for physiological conditions $(150 \mathrm{mM} \mathrm{NaCl})$ and compiled them in Table 1.

In step I, the dimer:tetramer interface slowly opens at the weaker $\alpha$-side into a metastable intermediate $\mathrm{O}_{\mathrm{cl}}^{\mathrm{MF} *}$ in rapid equilibrium with an open conformation $\mathrm{O}_{\mathrm{op}}^{\mathrm{HF}}$ through reversible disruption of the second dimer:tetramer interface (step II, tens of microseconds). There are several corroborating observations that the initial opening of the intact nucleosome (step I) is slow (>3 ms): (i) simulations for reproducing the FRET populations in the MFD diagrams with both FRET-lines connecting the static octasome $\mathrm{O}_{\mathrm{st}}^{\mathrm{MF}}$ and the dynamic octasome, $\mathrm{O}_{\mathrm{cl}}^{\mathrm{MF} *}$ and $\mathrm{O}_{\mathrm{op}}^{\mathrm{HF}}$, indicate that the opening rates at the $\alpha$-side must differ by factor $>1000$; (ii) this estimate agrees with a relaxation time of $5 \mathrm{~ms}$ reported by Lee et al. ${ }^{18,41}$. It is obvious that DNA breathing ${ }^{5,42}$ is essential for the motions of the histone core, which explains the complex kinetics with several additional relaxations times between 1 and $100 \mu$ s that is revealed by further analysis of the MFD data using filtered Fluorescence Correlation Spectroscopy (fFCS) (Supplementary Fig. 5 and Supplementary Note 4). While dynPDA is most sensitive to the internal opening dynamics in the time range $15-50 \mu$ s, fFCS monitors all FRET fluctuations in $\mathrm{I}_{\beta} \mathrm{I}_{\alpha}$ nucleosomes over four orders of magnitude in time (sub $\mu$ s to $\mathrm{ms}) \cdot \mathrm{O}_{\mathrm{op}}^{\mathrm{HF}}$ is reminiscent of the "butterfly" structure, originally sketched in our previous work ${ }^{14}$. In step III the $\alpha$-dimer dissociates, leading to a second intermediate, the dynamic hexasome with the states $\mathrm{H}_{\mathrm{cl}}^{\mathrm{MF} *}$ and $\mathrm{H}_{\mathrm{op}}^{\mathrm{HF}}$ (step IV) with slightly faster dynamics of the remaining dimer and partially unwrapped DNA (dominant species at $700 \mathrm{mM} \mathrm{NaCl}$ ). The final disassembly (step V) is the transition into tetrasomes $\mathrm{T}^{\mathrm{LF} / \mathrm{NF}}$ through loss of the second, the $\beta$-dimer. This last transition is slow (minutes to hours time scale) with rates comparable to those recently published by Plavner-Hazan et al. ${ }^{43}$ Note that at $\mathrm{NaCl}$ concentrations $>800 \mathrm{mM}$ tetrasomes are formed more easily (Supplementary Fig. 3). Tetrasome formation and subsequent tetrasome dissociation are both irreversible under single-molecule conditions (nM concentrations), in contrast to the stable equilibrium conditions at lower $\mathrm{NaCl}$ concentrations.

\section{Discussion}

The study of nucleosomes by salt-induced destabilization monitored by FRET and other biophysical techniques on the singlemolecule and ensemble level has been frequently applied to reveal their heterogeneity $11,12,14,17,36,42,44-53$. 


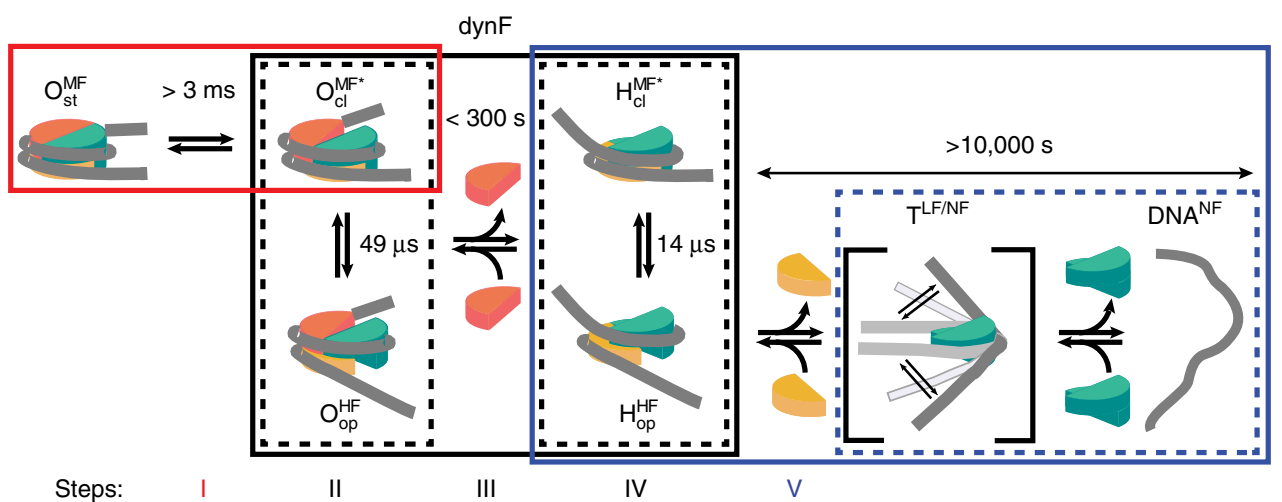

Fig. 8 Full kinetic scheme for nucleosome disassembly. At $150 \mathrm{mM} \mathrm{NaCl}$ concentration nucleosomes disassembly proceeds through the sequential loss of $\mathrm{H} 2 \mathrm{~A}-\mathrm{H} 2 \mathrm{~B}$ heterodimers as presented here with the corresponding relaxation times. The two heterodimers are shown in orange ( $\alpha$-side) and dark yellow ( $\beta$-side), while the tetramer is shown in turquoise. We resolve five steps in our smFRET experiments: (I) Disassembly of the intact nucleosome $\mathrm{O}_{\mathrm{st}}^{\mathrm{MF}}$ is predominantly initiated from the weaker binding $\alpha$-side, where opening of the first dimer:tetramer interface precedes dimer eviction $\mathrm{O}_{\mathrm{cl}}^{\mathrm{MF} *}$. (II-IV) The second dimer can reversibly detach from the tetramer once the first dimer:tetramer interface is broken $\mathrm{O}_{\mathrm{op}}^{\mathrm{HF}}$. The rate constant for the second dimer opening, however, is smaller when the first dimer is still present, probably due to stabilizing interactions between the dimers. Once the first dimer is gone $\mathrm{H}_{\mathrm{cl}}^{\mathrm{MF} *}$, reversible detachment $\mathrm{H}_{\mathrm{op}}^{\mathrm{HF}}$ and subsequent loss of the remaining dimer proceed at a faster rate. (V) The resulting tetrasome can adopt variable DNA geometries with most conformations leading to very low or no FRET ( $T^{\mathrm{LF} / N F}$ ). Note that at higher salt concentration the DNA can fully dissociate from the remaining tetramer $\left(\mathrm{DNA}^{\mathrm{NF}}\right)$. All corresponding kinetic and thermodynamic parameters are compiled in Table 1

Table 1 Kinetic and thermodynamic parameters of the nucleosome disassembly at $150 \mathrm{mM} \mathrm{NaCla}$

\begin{tabular}{|c|c|c|c|c|c|c|c|}
\hline \multirow{2}{*}{$\frac{\text { Step }}{1}$} & \multicolumn{2}{|c|}{ Rate constants } & \multicolumn{2}{|c|}{ Equilibrium constants } & \multicolumn{2}{|c|}{ Relaxation times } & \multirow{2}{*}{$\frac{\text { Methodb }}{\text { a, b (Figure 4) }}$} \\
\hline & 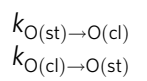 & $\begin{array}{l}<0.02 \times 10^{3} \mathrm{~s}^{-1} \\
<0.32 \times 10^{3} \mathrm{~s}^{-1}\end{array}$ & $K_{\mathrm{st} / \mathrm{cl}}^{\mathrm{O}}$ & $0.06 \pm 0.01^{c}$ & $t_{\mathrm{R}}^{\mathrm{O}(\mathrm{st} / \mathrm{cl})}$ & $>3 \mathrm{~ms}^{\mathrm{c}}$ & \\
\hline II & $\begin{array}{l}k_{\mathrm{O}(\mathrm{cl}) \rightarrow \mathrm{O}(\mathrm{op})} \\
\mathrm{k}_{\mathrm{O}(\mathrm{op}) \rightarrow \mathrm{O}(\mathrm{cl})}\end{array}$ & $\begin{array}{l}(13 \pm 1) 10^{3} \mathrm{~s}^{-1 \mathrm{c}} \\
(8.0 \pm 0.6) 10^{3} \mathrm{~s}^{-1}\end{array}$ & $\mathrm{~K}_{\mathrm{cl} / \mathrm{op}}^{\mathrm{O}}$ & $1.6 \pm 0.2$ & $t_{\mathrm{R}}^{\mathrm{O}(\mathrm{cl} / \mathrm{op})}$ & $(49 \pm 2) \mu \mathrm{s}$ & a (Figure 4, Supplementary Fig. 4) \\
\hline III & $\begin{array}{l}k_{\mathrm{O} \rightarrow \mathrm{H}} \\
k_{\mathrm{H} \rightarrow \mathrm{O}}\end{array}$ & $\begin{array}{l}>4 \times 10^{-3} \mathrm{~s}^{-1} \\
>10^{8} \mathrm{M}^{-1} \mathrm{~s}^{-1}\end{array}$ & $K_{\mathrm{dis}}^{\mathrm{O} / \mathrm{H}}$ & $(39 \pm 12) \mathrm{pM}^{\mathrm{c}}$ & $t_{\mathrm{R}}^{\mathrm{O} / \mathrm{H}}$ & $<300 \mathrm{~s}^{\mathrm{c}}, \mathrm{d}$ & a, c (Figure 4, Supplementary Fig. 3) \\
\hline IV & $\begin{array}{l}k_{\mathrm{H}(\mathrm{cl}) \rightarrow \mathrm{H}(\mathrm{op})} \\
k_{\mathrm{H}(\mathrm{op}) \rightarrow \mathrm{H}(\mathrm{cl})}\end{array}$ & $\begin{array}{l}(54 \pm 5) 10^{3} s^{-1 c} \\
(17 \pm 2) 10^{3} s^{-1 c}\end{array}$ & $K_{\mathrm{cl} / \mathrm{op}}^{\mathrm{H}}$ & $3.3 \pm 0.5$ & $t_{\mathrm{R}}^{\mathrm{H}(\mathrm{cl} / \mathrm{op})}$ & $(14 \pm 6) \mu \mathrm{s}$ & a (Figure 4, Supplementary Fig. 3) \\
\hline $\mathrm{V}$ & $\begin{array}{l}k_{\mathrm{H}(\mathrm{op}) \rightarrow \mathrm{T}} \\
k_{\mathrm{T} \rightarrow \mathrm{H}(\mathrm{op})}\end{array}$ & $\begin{array}{l}<(1.8 \pm 0.5) 10^{-5} \mathrm{~s}^{-1 \mathrm{c}} \\
<10^{7} \mathrm{M}^{-1} \mathrm{~s}^{-1 \mathrm{c}}\end{array}$ & $K_{\text {dis }}^{\mathrm{H} / \mathrm{T}}$ & $>2 \mathrm{pM}$ & $t_{\mathrm{R}}^{\mathrm{H} / \mathrm{T}}$ & $>10,000 s^{c, d, e}$ & c (Supplementary Fig. 2) \\
\hline \multicolumn{8}{|c|}{$\begin{array}{l}\text { aThe reaction scheme and the nucleosome species are displayed in Fig. } 8 \text { with the following characterization: }(\mathrm{O}) \text { octasome, (H) hexasome, (T) tetrasome; (st) static closed, (cl) dynamic closed, (op) } \\
\text { dynamic open } \\
\text { bMethods: (a) dynPDA, (b) simulation, (c) trace analysis } \\
\text { 'Directly determined parameters } \\
\text { dEstimated for }[\mathrm{H} 2 \mathrm{~A}-\mathrm{H} 2 \mathrm{~B}] \approx 10 \mathrm{pM} \\
\text { eNote that at } \mathrm{NaCl} \text { concentrations }>800 \mathrm{mM} \text { tetrasomes (Supplementary Fig. } 3 \text { ) are formed more easily. In the last step DNA dissociates irreversibly under single-molecule conditions }\end{array}$} \\
\hline
\end{tabular}

In recent smFRET experiments ${ }^{43}$, salt-induced dissociation kinetics were measured on a minute-to-hour time scale at 5 and $300 \mathrm{mM} \mathrm{NaCl}$ and $3 \mathrm{pM}$ nucleosome concentration. While these conditions spanned the physiological salt range, the kinetics could only describe the slow transition from the intact to the partly dissociated nucleosome (the $\mathrm{T}^{\mathrm{LF} / \mathrm{NF}}$ state) without additional resolved intermediates. Recently, Chen et al. studied nucleosome disassembly kinetics in very high salt (up to $1.9 \mathrm{M}$ $\mathrm{NaCl}$ ) by stopped-flow with small-angle X-ray scattering (SAXS) and ensemble FRET detection. The development of the nucleosomal ensemble over time led to postulating unwrapping states with transition kinetics in the range of 50 milliseconds to seconds. Due to the reduced data quality in fast time-resolved SAXS, structural predictions from such experiments are necessarily limited in their precision. Therefore, we applied smFRET with MFD, dynPDA, fFCS and detailed analysis for potential structural species to gain an in-depth view of nucleosome disassembly at arbitrary salt concentrations and short time scales. We revealed complex kinetics with fast exchanging initially opened states (with a dominating relaxation time of tens of microseconds), in which the nucleosome internal structure is loosened and the DNA is still wrapped but potentially breathing ${ }^{5,42}$. We suggest a multistep kinetic scheme that provides details of nucleosome assembly and disassembly and critically depends on the overall nucleosome concentration. In the following, we discuss four important observations.

1. Nucleosome dissociation begins with DNA unwrapping. Free in solution, the 601 nucleosomes preferentially open up from one side, where the DNA is more loosely bound. We show for hexasomes that further disassembly starts with sequencedependent DNA unwrapping in the entry-exit region at the $\alpha$ side and detect additional kinetic transitions $(\sim 0.1 \mathrm{~ms})$ by fFCS While asymmetric DNA unwrapping in surface-attached nucleosomes under tension was reported by $\mathrm{Ngo}$ et al. ${ }^{34}$, no direct evidence existed for nucleosomes without external tension in solution. Ngo et al. reported that the DNA half whose central region contains multiple TA-steps at $\sim 10$ bp spacing (our " $\beta$ side") is more resilient against mechanical unwrapping than the weaker binding DNA side. This agrees with our observations that 
local flexibility of DNA controls nucleosome stability directly, even without external tension.

2. Nucleosome dynamics is linked with $\mathrm{H} 2 \mathrm{~A}-\mathrm{H} 2 \mathrm{~B}$ dimer exchange. The correlation of DNA stiffness and H2A-H2B dimer eviction supports earlier in vitro observations that the transcription barrier formed by the nucleosome is polar ${ }^{54}$. However, this hypothesis is still controversial: a recent study ${ }^{55}$ did not find an orientation-dependent difference in transcription rates through 601 nucleosomes, implying that factors beyond DNA sequence must play a role ${ }^{56,57}$. The fast dynamic transition shown here agrees with our earlier finding ${ }^{14}$ that before dimer eviction, the dimer:tetramer interface is disrupted transiently. A cleft, some $\mathrm{nm}$ in size, within the histone core could then serve as a recognition motif for typical nuclear proteins. DNA-histone interactions are clustered in 14 discrete regions along the superhelical DNA path, where the average binding energy per cluster only slightly exceeds thermal energy ${ }^{2,58}$. In an octasome without external tension, DNA would unpeel by successive disruption of DNA-histone interactions from the DNA ends, exposing DNA inside the nucleosome much less frequently than in the entry/exit region $^{6}$. After opening of the dimer:tetramer interface, as supposed in our model, DNA-histone contacts adjacent to the interface are no longer shielded from disruption, offering much easier access.

3. H2A-H2B dimers are step-wisely released. Earlier on we had proposed a step-wise disassembly through loss of $\mathrm{H} 2 \mathrm{~A}-\mathrm{H} 2 \mathrm{~B}$ dimers $^{12}$, but the lack of analytical tools at this time precluded the detailed kinetic and structural finding that the dimer:tetramer interface is crucial for nucleosome stability and it must already open reversibly ${ }^{14}$ in the dynamic octasome to prime the system for subsequent dimer eviction. Considering $a \approx 20^{\circ}$ opening of the intact nucleosome (species $\mathrm{MF}$ ), our geometric model predicts a distance change of $\approx 4 \AA(\Delta E \approx 0.1)$ that approximately corresponds to the FRET efficiency of $\mathrm{MF}^{\star}$ (Fig. 7).

Interactions between $\mathrm{H} 2 \mathrm{~A}-\mathrm{H} 2 \mathrm{~B}$ dimers through hydrogen bonds between their L1 loop domains ${ }^{1}$ stabilize the nucleosome, increasing the energetic barrier for the opening of the first dimer. Our structure-based finding that the initial opening might be significantly slower for the first than for the second dimer, nicely agrees with our observed millisecond and microsecond dynamics for the corresponding process (steps I and II $\left(\mathrm{O}_{\mathrm{cl}}^{\mathrm{MF} *} \rightleftarrows \mathrm{O}_{\mathrm{op}}^{\mathrm{HF}}\right)$ and IV in Table 1, respectively). Earlier ensemble FRET experiments suggested such a cooperativity ${ }^{59}$, but by monitoring the dynamic octasome and hexasome intermediates it could be detected in this study.

Arimura et al. reported SAXS data that suggested an overall similar static core structure of hexasomes and intact nucleosomes ${ }^{60}$. Here, however, we found the hexasome state as part of the dynamic FRET population. Hexasomes are also highly dynamic entities undergoing rapid transitions (tens of microseconds) between open and closed histone core conformations. To our best knowledge, this constitutes the first report of such fast dynamics in hexasomes.

We finally note that the energetic barrier for the release of the first heterodimer could in principal also be lowered by nucleosome gaping, where the two (H2A-H2B)/(H3-H4) halves of the octamer transiently open-up in a clam-shell like motion $^{28,29}$. Reversible opening of the dimer:tetramer interface could well occur in the "gaped" conformation, after disruption of the stabilizing interactions between the L1 loops. This is, however, not a likely candidate for $\mathrm{MF}^{\star}$ and $\mathrm{HF}$, since the preceding MF$\mathrm{MF}^{\star}$ transition exhibits strong concentration dependence, that is indicative for a dissociation process. Gaping as described by Ngo et $\mathrm{al}^{29}$, on the other hand, would not depend on nucleosome concentration.

4. Tetrasome disassembly. Finally, the second histone dimer dissociates, followed by full opening of the DNA and $(\mathrm{H} 3-\mathrm{H} 4)_{2}$ tetramer release. It is noteworthy that in the studies of ${ }^{14}$ we observed full tetramer release almost simultaneously with full DNA unfolding already at $1.2 \mathrm{M}$ salt ${ }^{14}$, while Chen et al. ${ }^{30}$ used $1.9 \mathrm{M}$ salt to reach this limit. This could imply different opening mechanisms in high and low salt or simply caused by the nonequilibrium conditions inherent to stopped flow experiments reported in reference ${ }^{30}$.

In conclusion, our results provide important insights into mononucleosome dynamics which could be more representative for terminal ${ }^{61}$ than internal sites ${ }^{20}$ in nucleosome arrays used as models for chromatin fibers ${ }^{62}$. MFD enabled us to observe octasome and hexasome dynamics in reversible opening of the dimer:tetramer interface. Further analysis of the concentration and salt dependencies allows us to postulate a minimum kinetic scheme for the nucleosome structure opening through at least seven species from the compact mononucleosome to the free DNA. Moreover, the methodology developed in this work paves the way to systematic investigations of the role of histone tail modifications and interactions in chromatin structure, enabling us to develop a detailed dynamic picture of this central biological entity.

\section{Methods}

Preparation of mononucleosomes. Mononucleosomes were made from 170 bp DNA fragments containing the Widom 601 positioning sequence ${ }^{32}$ and Xenopus laevis recombinant histones. DNA was prepared by PCR with fluorescent primers (IBA) and purified on Gen-Pak FAX HPLC (Waters). Recombinant wild type (wt) histones were expressed in E. coli bacteria and purified by gel filtration ${ }^{42}$. For some experiments we mutated histone H2A, replacing arginine at position 81 with alanine (H2A-R81A). H2B-T112C mutants for histone dimer labeling were purchased from Planet Protein (Colorado State University).

Histone octamers were prepared by mixing histones in unfolding buffer $(7 \mathrm{M}$ Guanidine) followed by dialysis against high salt $(2 \mathrm{M} \mathrm{NaCl}$ in TE buffer: $10 \mathrm{mM}$ Tris- $\mathrm{HCl}$ containing $0.1 \mathrm{mM}$ EDTA, $\mathrm{pH}=7.5$ ). Octamers in best proportion were selected after size exclusion FPLC (Superdex 200HR 10/10) and Triton X-100/ acetic acid/urea (TAU) gel analysis.

In single color excitation experiments, FRET donor and acceptor were Alexa488 (A488) and Alexa594 (A594). In MFD-PIE, Cy5 was used as acceptor. The acceptor was attached to the DNA through amino-C6 linker either internally about half a turn away from the dyad axis $\left(\mathrm{I}_{\beta}\right)$ or near the dyad axis at either $\alpha$ - or $\beta$-side (Dy or $\left.\mathrm{Dy}_{\beta}\right)$. Note that according to the nucleosome crystal structure the dyad is localized at around $-6 \mathrm{bp}$ of our sequence ${ }^{63}$. The donor was attached to DNA via amino-C6 linker at one or the other end $\left(\mathrm{E}_{\alpha}\right.$ or $\left.\mathrm{E}_{\beta}\right)$, at an internal position about half a turn away from the dyad axis $\left(\mathrm{I}_{\beta}\right)$ or bound to histone $\mathrm{H} 2 \mathrm{~B}$ at position 112 using the H2B-T112C mutant ${ }^{14}$. Five types of nucleosomes were prepared for FRET measurements (Table 2, see also Supplementary Methods):

Table 2 Notation of nucleosome constructs

\section{Notation Sample description}

$\mathrm{I}_{\beta} \mathrm{l}_{\alpha}$-wt DNA labeled at +41 (A488) and -53 (A594) and wt octamers

$\mathrm{I}_{\beta} \mathrm{I}_{\alpha}$-mut DNA labeled at +41 (A488) and -53 (A594) and octamers containing H2A-R81A mutated histones

$\mathrm{E}_{\beta} \mathrm{Dy}_{\alpha} \quad$ DNA labeled at $5^{\prime}$ end $\beta\left(\mathrm{E}_{\beta}\right)$ with $\mathrm{A} 488$ and at position -15 (close to the dyad) on the other strand (Dy ${ }_{\alpha}$ ) with $A 594$, plus wt octamers

$\mathrm{E}_{\alpha} \mathrm{Dy}_{\beta} \quad$ Analogous to nucleosome $E_{\beta} D y_{\alpha}$ with DNA label positions on the complementary strands (A488 at $5^{\prime}$ end $\alpha, A 594$ at +15 )

$\mathrm{H} 2 \mathrm{~B}-\mathrm{Dy} \mathrm{y}_{\alpha} \quad$ DNA labeled at -15 (near the dyad) with Cy5 and octamers containing H2B-A488 
In $\mathrm{I}_{\beta} \mathrm{I}_{\alpha}$ constructs the dyes were placed at the heterodimer-binding region of nucleosomal DNA, such that their local environment is sensitive to the presence or absence of H2A-H2B heterodimers.

DNA labeling efficiency was $>95 \%$ as measured by absorption spectroscopy and by single-molecule fluorescence with alternating laser excitation. Labeling efficiency for histone $\mathrm{H} 2 \mathrm{~B}$ was $~ 45 \%$ as determined from TAU gel analysis and spectroscopic measurements. Nucleosomes were prepared by mixing DNA and histone octamers in TE buffer at high salt $(2 \mathrm{M} \mathrm{NaCl}$ ), then slow salt dialysis (mini-dialyzing tubes, cut off $7 \mathrm{kDa}$, Pierce) down to $5 \mathrm{mM}$. Molar DNA:Octamer ratios in the range 1:1.5 - 1:1.8 were adjusted such that $<5 \%$ free DNA was visible after reconstitution. Donor-only labeled nucleosomes, free double labeled DNA and donor- or acceptor-only labeled DNA were prepared for control experiments from the same sources. For some experiments the overall nucleosome concentration was adjusted by adding unlabeled nucleosomes prepared with the same octamers. The quality of nucleosome reconstitution was tested by absorption spectroscopy for detecting possible aggregates and by PAA gel electrophoresis (6\%, 60:1 acrylamide: bisacrylamide). Residual aggregates were removed by centrifugation (Eppendorf Centrifuge $5417 \mathrm{R}$ ) at $10,000 \mathrm{rpm}$ and $10^{\circ} \mathrm{C}$ for $10 \mathrm{~min}$. Ensemble fluorescence anisotropy of the attached dyes was below $r=0.2$ in DNA and nucleosome samples, indicating sufficient dye mobility. Nucleosomes with concentrations above $100 \mathrm{nM}$ were stable at $4^{\circ} \mathrm{C}$ for several weeks.

Ensemble FRET measurements. Ensemble FRET experiments were performed on the microplate reader, Typhoon multimode imager ${ }^{36}$. A freshly cleaned and passivated 384-well microplate was loaded with 300 or $600 \mathrm{pM}$ labeled nucleosomes that had been incubated for $60 \mathrm{~min}$ at $\mathrm{NaCl}$ concentrations ranging from 5 to $1200 \mathrm{mM}$. To quantify FRET, the donor and acceptor fluorescence were recorded at $488 \mathrm{~nm}$ excitation using bandpass filters provided in the instrument (donor channel $\left(I_{\mathrm{D}}{ }^{\text {Dex }}\right)$ : 520BP40, acceptor channel $\left(I_{\mathrm{A}}{ }^{\text {Dex }}\right)$ : 610BP30). An additional scan of the acceptor signal at $532 \mathrm{~nm}$ excitation $\left(I_{\mathrm{A}}\right.$ Aex $)$ was used to account for direct acceptor excitation. All control samples (buffer only, donor-only, acceptor-only and double labeled DNA fragments with no FRET) were measured in parallel to the actual nucleosome samples. For each sample, the proximity ratio $(P)$ was computed from the measured fluorescence intensities considering calculated correction factors and averaged over three independent wells:

$$
P=\frac{\left(I_{\mathrm{A}}^{\text {Dex }}\right)_{\text {corr }}}{\left(I_{\mathrm{D}}^{\text {Dex }}\right)_{\text {corr }}+\left(I_{\mathrm{A}}^{\text {Dex }}\right)_{\text {corr }}}
$$

where $(I \ldots)_{\text {corr }}$ represent corrected fluorescence intensities as described in Supplementary Note 5 .

Data analysis. Ensemble $P$ data were normalized to a scale of $0 \%$ (no FRET) to $100 \%$ (maximum FRET) and approximated by a sigmoidal function (Supplementary Note 5, Supplementary Equation 36 ) to obtain the salt concentration at which the proximity ratio dropped to half of its maximal value ( $c_{1 / 2}$ value). This value was taken as a measure for the stability of the corresponding FRET pair ${ }^{36}$.

Single-molecule FRET experiments. MFD experiments were carried out with a confocal epi-illuminated setup based on an Olympus IX70 inverted microscope as described in refs. ${ }^{31,64-67}$. In brief, a linearly polarized pulsed $495 \mathrm{~nm}$ diode laser (LDH-D-C 495, PicoQuant; $36 \mu \mathrm{W}$ in the sample) operating at $64 \mathrm{MHz}$ was focused into the sample solution by a $60 \times / 1.2 \mathrm{NA}$ water immersion objective (UPLAPO 60x, Olympus, Germany). The photon train was divided into its parallel and perpendicular components by a polarizing beamsplitter cube (VISHT11, Gsänger) and then into spectral ranges below and above $595 \mathrm{~nm}$ by a dichroic beamsplitter (595 LPXR, AHF). Additionally, red (HQ 645/75 nm for Alexa594) and green (HQ 533/46 nm for Alexa488) bandpass filters (both provided by AHF) in front of the detectors ensured that only fluorescence from the acceptor and donor molecules were registered, while residual laser light and Raman scattering from the solvent were blocked. Photons were detected by four avalanche photodiodes (green channels: $\tau$-SPAD-100, PicoQuant; red channels: SPCM-AQR-14, Perkin Elmer). The signals from all detectors were guided through a passive delay unit and two routers to two synchronized time-correlated single photon counting boards (SPC 132, Becker \& Hickl) that were connected to a PC. The high frequency output of the diode laser serves as the common time base for synchronization (TCSPC stop signal) and the macro time counter for both SPC-132 counting boards. Bursts of fluorescence photons are distinguished from the background of $0.3-0.4 \mathrm{kHz}$ by applying certain threshold intensity criteria ${ }^{64}$. For analysis, several parameters including fluorescence lifetime, anisotropy, and FRET efficiency were computed per burst to classify the molecules according to multidimensional relations between these parameters. Further details are given in Supplementary Methods.

MFD measurements with pulsed interleaved excitation (PIE) were performed employing a setup similar to the one described above. In PIE measurements ${ }^{22}$, donor and acceptor are sequentially excited by rapidly alternating laser pulses. MFD can be performed on both dyes, allowing computation of the donor-acceptor ratio (stoichiometry, $\mathrm{S}_{\mathrm{PIE}}$ ) for each particle. Excitation is achieved using $485 \mathrm{~nm}$ and 635 nm pulsed diode lasers (LDH-D-C 485 and LDH-P-C-635B, respectively; both PicoQuant, operated at $32 \mathrm{MHz}$ ) on an Olympus IX71 microscope. Laser power in the sample was 34 and $7 \mu \mathrm{W}$, respectively. After separating the fluorescence signal according to color and polarization, each of the four channels was split again using $50 / 50$ beam splitters in order to get dead time free fFCS curves, resulting in a total of eight detection channels. Detector types for green and red channels were the same as described above, bandpass filters were HQ 520/35 and HQ 720/150 (both AHF) for green and red channels, respectively. The detector outputs were recorded by a TCSPC module (HydraHarp 400, PicoQuant) and stored on a PC. Analysis of the single photon data stream was done using threshold criteria ${ }^{22}$

Measurement conditions for ensemble and smFRET experiments. For experiments nucleosomes were freshly diluted into $0.02 \mu \mathrm{m}$-filtered measuring buffer (TE containing $\mathrm{NaCl}$ as noted), $1 \mathrm{mM}$ ascorbic acid (Sigma-Aldrich) and $0.01 \%$ Nonidet P40 (Roche Diagnostics) and unlabeled nucleosomes as noted. 50-100 $\mu \mathrm{l}$ of nucleosomes were placed into 384-well microplates (SensoPlate Plus, Greiner BioOne) that had been passivated with Sigmacote (Sigma-Aldrich). In ensemble FRET experiments the passivated microplate was loaded with 300 or $600 \mathrm{pM}$ labeled nucleosomes that had been incubated for $60 \mathrm{~min}$ at $\mathrm{NaCl}$ concentrations ranging from 5 to $1200 \mathrm{mM}$. If not otherwise stated, in single-molecule FRET experiments $20 \mathrm{pM}$ of labeled nucleosomes were used. Where necessary, an appropriate amount of unlabeled nucleosomes was then added to adjust overall nucleosome concentration. Data were taken for at least $90 \mathrm{~min}$ per sample. Series of measurements, e.g. salt titration or nucleosome titration, were repeated and results of the respective analyses averaged. Due to slight day-to-day variations in the sample preparations only complete series were used for averaging. Displayed error bars reflect these variations.

Determination of FRET-based interdye distances. To confirm our FRET-based identification of structural substates for the construct $\mathrm{H} 2 \mathrm{~B}-\mathrm{Dy}_{\alpha}$, we measured donor mobility via its burstwise anisotropy $r_{\mathrm{D}}$ (Supplementary Fig. 1b, Supplementary Table 4 , Supplementary Note 6 ) to validate the used $R_{0}$, assuming a FRET orientation factor $\kappa^{2}=2 / 3$ for the isotropic average. Each population was well described by a single donor anisotropy value $\left(\operatorname{dynF}\left(r_{\mathrm{D}}=0.135\right), \mathrm{MF}\left(r_{\mathrm{D}}=0.114\right)\right.$, and $\left.\operatorname{LF}\left(r_{\mathrm{D}}=0.080\right)\right)$, decreasing with increasing lifetime. The anisotropies of all FRET species are in agreement with high mobility (average rotational correlation time $\rho \approx 1 \mathrm{~ns}$ ), so that $\kappa^{2}=2 / 3$ can be safely assumed.

For the five nucleosome constructs we employed two FRET pairs with the following Förster radii: $R_{0}($ Alexa488 - Cy5 $)=52.0 \AA$ and $R_{0}($ Alexa488 - Alexa594 $)=$ $55.6 \AA$. The crystal structure of mononucleosome (PDB ID: 3LZ1) was used as template for the interdye distance prediction (for details (geometric description, computer code and comparison with earlier work ${ }^{11}$ see Supplementary Note 3). The dye distribution was modeled by the accessible volume approach (AV). Here donor and acceptor fluorophores are approximated by a sphere with an empirical radius of $R_{\text {dye, }}$ where the central atom of the fluorophore is connected by a flexible linkage of a certain effective length $L_{\text {link }}$ and width $w_{\text {link }}$. All geometric parameters for the dyes were: Alexa488 and Alexa594: $L_{\text {link }}=20 \AA$, $w_{\text {link }}=4.5 \AA, R_{\text {dye }}=1.5 \AA$; Cy5: $L_{\text {link }}=22 \AA, w_{\text {link }}=4.5 \AA, R_{\text {dye }}=3.5 \AA$.

For better direct comparability, we convert average distances from different data-sources, i.e. mean distance, $\left\langle R_{\mathrm{DA}}\right\rangle$, from fluorescence lifetime analysis; FRET averaged distance, $\left\langle R_{\mathrm{DA}}\right\rangle_{\mathrm{E}}$, from intensity analysis by PDA and distances between the mean positions of donor and acceptor, $R_{\mathrm{mp}}$, from geometric modeling (Supplementary Note 3 ) to $\left\langle R_{\mathrm{DA}}\right\rangle_{\mathrm{E}}$; e.g., $\left\langle R_{\mathrm{DA}}\right\rangle$ is converted to FRET-average mean distance $\left\langle R_{\mathrm{DA}}\right\rangle_{\mathrm{E}}$ by the following polynomial: $\left\langle R_{\mathrm{DA}}\right\rangle_{\mathrm{E}}=15.3230+0.592830$ $\left\langle R_{\mathrm{DA}}\right\rangle+0.00175370\left\langle R_{\mathrm{DA}}\right\rangle^{2}-0.0000032013\left\langle R_{\mathrm{DA}}\right\rangle^{3}$. The coefficients of the other polynomials are compiled in Supplementary Table 1.

Code availability. Most general custom-made computer code is directly available from http://www.mpc.hhu.de/en/software. The source code for simulating the curves in Fig. 7 using the programming tool IGOR Pro (WaveMetrics, Lake Oswego, OR, USA) is provided in Supplementary Note 3. Additional computer code custom-made for this publication is available upon request from the corresponding authors.

\section{Data availability}

Other data supporting the findings in this work are available from the corresponding authors upon reasonable request.

Received: 17 March 2017 Accepted: 17 September 2018 Published online: 06 November 2018

\section{References}

1. Luger, K., Mäder, A. W., Richmond, R. K., Sargent, D. F. \& Richmond, T. J. Crystal structure of the nucleosome core particle at 2.8 A resolution. Nature 389, 251-260 (1997). 
2. Davey, C. A., Sargent, D. F., Luger, K., Maeder, A. W. \& Richmond, T. J. Solvent mediated interactions in the structure of the nucleosome core particle at 1.9 a resolution. J. Mol. Biol. 319, 1097-1113 (2002).

3. Aoyagi, S. et al. Nucleosome remodeling by the human SWI/SNF complex requires transient global disruption of histone-DNA interactions. Mol. Cell. Biol. 22, 3653-3662 (2002).

4. Li, G., Levitus, M., Bustamante, C. \& Widom, J. Rapid spontaneous accessibility of nucleosomal DNA. Nat. Struct. Mol. Biol. 12, 46-53 (2005).

5. Li, G. \& Widom, J. Nucleosomes facilitate their own invasion. Nat. Struct. Mol. Biol. 11, 763-769 (2004).

6. Tims, H. S., Gurunathan, K., Levitus, M. \& Widom, J. Dynamics of nucleosome invasion by DNA binding proteins. J. Mol. Biol. 411, 430-448 (2011).

7. Clark, D. J. \& Felsenfeld, G. A nucleosome core is transferred out of the path of a transcribing polymerase. Cell 71, 11-22 (1992).

8. Lovullo, D., Daniel, D., Yodh, J., Lohr, D. \& Woodbury, N. W. A fluorescence resonance energy transfer-based probe to monitor nucleosome structure. Anal. Biochem. 341, 165-172 (2005).

9. Koopmans, W. J., Brehm, A., Logie, C., Schmidt, T. \& van Noort, J. Single-pair FRET microscopy reveals mononucleosome dynamics. J. Fluoresc. 17, 785-795 (2007).

10. Blosser, T. R., Yang, J. G., Stone, M. D., Narlikar, G. J. \& Zhuang, X. Dynamics of nucleosome remodelling by individual ACF complexes. Nature 462, 1022-1027 (2009).

11. Gansen, A., Tóth, K., Schwarz, N. \& Langowski, J. Structural variability of nucleosomes detected by single-pair Förster resonance energy transfer: Histone acetylation, sequence variation, and salt effects. J. Phys. Chem. B 113, 2604-2613 (2009).

12. Gansen, A. et al. Nucleosome disassembly intermediates characterized by single-molecule FRET. Proc. Natl. Acad. Sci. USA 106, 15308-15313 (2009)

13. Koopmans, W. J., Buning, R., Schmidt, T. \& van Noort, J. spFRET using alternating excitation and FCS reveals progressive DNA unwrapping in nucleosomes. Biophys. J. 97, 195-204 (2009).

14. Böhm, V. et al. Nucleosome accessibility governed by the dimer/tetramer interface. Nucleic Acids Res. 39, 3093-3102 (2011).

15. Chen, J., Miller, A., Kirchmaier, A. L. \& Irudayaraj, J. M. Single-molecule tools elucidate H2A.Z nucleosome composition. J. Cell Sci. 125, 2954-2964 (2012).

16. Tóth, K. et al. Histone- and DNA sequence-dependent stability of nucleosomes studied by single-pair FRET. Cytometry A. 83, 839-846 (2013).

17. Gansen, A., Tóth, K., Schwarz, N. \& Langowski, J. Opposing roles of H3- and H4-acetylation in the regulation of nucleosome structure--a FRET study. Nucleic Acids Res. 43, 1433-1443 (2015).

18. Kim, J., Wei, S., Lee, J., Yue, H. \& Lee, T. H. Single-molecule observation reveals spontaneous protein dynamics in the nucleosome. J. Phys. Chem. B 120, 8925-8931 (2016).

19. Poirier, M. G., Oh, E., Tims, H. S. \& Widom, J. Dynamics and function of compact nucleosome arrays. Nat. Struct. Mol. Biol. 16, 938 (2009).

20. Kilic, S. et al. Single-molecule FRET reveals multiscale chromatin dynamics modulated by HP1 alpha. Nat. Commun. 9, 235 (2018).

21. Felekyan, S., Sanabria, H., Kalinin, S., Kühnemuth, R. \& Seidel, C. A. M Analyzing Förster resonance energy transfer with fluctuation algorithms. Methods Enzymol. 519, 39-85 (2013).

22. Kudryavtsev, V. et al. Combining MFD and PIE for accurate single-pair Förster resonance energy transfer measurements. ChemPhysChem 13, 1060-1078 (2012).

23. Kalinin, S. et al. A toolkit and benchmark study for FRET-restrained highprecision structural modeling. Nat. Methods 9, 1218-1225 (2012).

24. Felekyan, S., Kalinin, S., Sanabria, H., Valeri, A. \& Seidel, C. A. M. Filtered FCS: species auto- and cross-correlation functions highlight binding and dynamics in biomolecules. ChemPhysChem 13, 1036-1053 (2012).

25. Cristovao, M. et al. Single-molecule multiparameter fluorescence spectroscopy reveals directional MutS binding to mismatched bases in DNA. Nucleic Acids Res. 40, 5448-5464 (2012).

26. Sindbert, S. et al. Accurate distance determination of nucleic acids via Förster resonance energy transfer: implications of dye linker length and rigidity. $J$. Am. Chem. Soc. 133, 2463-2480 (2011).

27. Chen, Y. et al. Revealing transient structures of nucleosomes as DNA unwinds. Nucleic Acids Res. 42, 8767-8776 (2014).

28. Schlingman, D. J., Mack, A. H., Kamenetska, M., Mochrie, S. G. \& Regan, L. Routes to DNA accessibility: alternative pathways for nucleosome unwinding. Biophys. J. 107, 384-392 (2014).

29. Ngo, T. T. \& Ha, T. Nucleosomes undergo slow spontaneous gaping. Nucleic Acids Res. 43, 3964-3971 (2015).

30. Chen, Y. et al. Asymmetric unwrapping of nucleosomal DNA propagates asymmetric opening and dissociation of the histone core. Proc. Natl. Acad. Sci. USA 114, 334-339 (2017).
31. Widengren, J. et al. Single-molecule detection and identification of multiple species by multiparameter fluorescence detection. Anal. Chem. 78, 2039-2050 (2006).

32. Lowary, P. T. \& Widom, J. New DNA sequence rules for high affinity binding to histone octamer and sequence-directed nucleosome positioning. J. Mol. Biol. 276, 19-42 (1998).

33. Lerner, E. et al. Toward dynamic structural biology: two decades of singlemolecule Förster resonance energy transfer. Science 359, eaan1133 (2018).

34. Ngo, T. T., Zhang, Q., Zhou, R., Yodh, J. G. \& Ha, T. Asymmetric unwrapping of nucleosomes under tension directed by DNA local flexibility. Cell 160, 1135-1144 (2015).

35. Lee, N. K. et al. Accurate FRET measurements within single diffusing biomolecules using alternating-laser excitation. Biophys. J. 88, 2939-2953 (2005).

36. Gansen, A., Hieb, A. R., Böhm, V., Tóth, K. \& Langowski, J. Closing the gap between single molecule and bulk FRET analysis of nucleosomes. PLOS ONE $\mathbf{8}$, e57018 (2013)

37. Kalinin, S., Valeri, A., Antonik, M., Felekyan, S. \& Seidel, C. A. M. Detection of structural dynamics by FRET: A photon distribution and fluorescence lifetime analysis of systems with multiple states. J. Phys. Chem. B 144, 7983-7995 (2010).

38. Kalinin, S., Felekyan, S., Antonik, M. \& Seidel, C. A. M. Probability distribution analysis of single-molecule fluorescence anisotropy and resonance energy transfer. J. Phys. Chem. B 111, 10253-10262 (2007).

39. Biswas, M., Voltz, K., Smith, J. C. \& Langowski, J. Role of histone tails in structural stability of the nucleosome. PLoS Comput. Biol. 7, e1002279 (2011).

40. Lehmann, K. et al. Effects of charge-modifying mutations in histone H2A a 3 domain on nucleosome stability assessed by single-pair FRET and MD simulations. Sci. Rep. 7, 13303 (2017).

41. Wei, S. J., Falk, S. J., Black, B. E. \& Lee, T. H. A novel hybrid single molecule approach reveals spontaneous DNA motion in the nucleosome. Nucleic Acids Res. 43, E111-U148 (2015).

42. Tóth, K., Brun, N. \& Langowski, J. Chromatin compaction at the mononucleosome level. Biochemistry 45, 1591-1598 (2006).

43. Hazan, N. P. et al. Nucleosome core particle disassembly and assembly kinetics studied using single-molecule fluorescence. Biophys. J. 109, 1676-1685 (2015)

44. Yager, T. D., McMurray, C. T. \& van Holde, K. E. Salt-induced release of DNA from nucleosome core particles. Biochemistry 28, 2271-2281 (1989).

45. Ballestar, E. \& Franco, L. Use of the transglutaminase reaction to study the dissociation of histone $\mathrm{N}$-terminal tails from DNA in nucleosome core particles. Biochemistry 36, 5963-5969 (1997).

46. Khrapunov, S. N., Dragan, A. I., Sivolob, A. V. \& Zagariya, A. M. Mechanisms of stabilizing nucleosome structure. Study of dissociation of histone octamer from DNA. Biochim. Biophys. Acta 1351, 213-222 (1997).

47. Ballestar, E., Boix-Chornet, M. \& Franco, L. Conformational changes in the nucleosome followed by the selective accessibility of histone glutamines in the transglutaminase reaction: effects of ionic strength. Biochemistry $\mathbf{4 0}$, 1922-1929 (2001)

48. Park, Y. J., Dyer, P. N., Tremethick, D. J. \& Luger, K. A new fluorescence resonance energy transfer approach demonstrates that the histone variant $\mathrm{H} 2 \mathrm{AZ}$ stabilizes the histone octamer within the nucleosome. J. Biol. Chem. 279, 24274-24282 (2004).

49. Bertin, A., Leforestier, A., Durand, D. \& Livolant, F. Role of histone tails in the conformation and interactions of nucleosome core particles. Biochemistry 43 4773-4780 (2004)

50. Gansen, A., Hauger, F., Tóth, K. \& Langowski, J. Single-pair fluorescence resonance energy transfer of nucleosomes in free diffusion: optimizing stability and resolution of subpopulations. Anal. Biochem. 368, 193-204 (2007).

51. Kepert, J. F. et al. Conformation of reconstituted mononucleosomes and effect of linker histone $\mathrm{H} 1$ binding studied by scanning force microscopy. Biophys. J. 85, 4012-4022 (2003).

52. Hammermann, M. et al. Salt-dependent compaction of di- and trinucleosomes studied by small- angle neutron scattering. Biophys. J. 79, 584-594 (2000).

53. Tóth, K., Brun, N. \& Langowski, J. Trajectory of nucleosomal linker DNA studied by fluorescence resonance energy transfer. Biochemistry 40, 6921-6928 (2001)

54. Bondarenko, V. A. et al. Nucleosomes can form a polar barrier to transcript elongation by RNA polymerase II. Mol. Cell 24, 469-479 (2006).

55. Perales, R., Zhang, L. \& Bentley, D. Histone occupancy in vivo at the 601 nucleosome binding element is determined by transcriptional history. Mol. Cell. Biol. 31, 3485-3496 (2011).

56. Chakraborty, S. A., Kazi, A. A., Khan, T. M. \& Grigoryev, S. A. Nucleosomepositioning sequence repeats impact chromatin silencing in yeast minichromosomes. Genetics 198, 1015-1029 (2014). 
57. Gaykalova, D. A. et al. A polar barrier to transcription can be circumvented by remodeler-induced nucleosome translocation. Nucleic Acids Res. 39, 3520-3528 (2011).

58. Wocjan, T., Klenin, K. \& Langowski, J. Brownian dynamics simulation of DNA unrolling from the nucleosome. J. Phys. Chem. B 113, 2639-2646 (2009).

59. Hoch, D. A., Stratton, J. J. \& Gloss, L. M. Protein-protein Förster resonance energy transfer analysis of nucleosome core particles containing H2A and H2A.Z. J. Mol. Biol. 371, 971-988 (2007).

60. Arimura, Y., Tachiwana, H., Oda, T., Sato, M. \& Kurumizaka, H. Structural analysis of the hexasome, lacking one histone $\mathrm{H} 2 \mathrm{~A} / \mathrm{H} 2 \mathrm{~B}$ dimer from the conventional nucleosome. Biochemistry 51, 3302-3309 (2012).

61. Fierz, B., Kilic, S., Hieb, A. R., Luger, K. \& Muir, T. W. Stability of nucleosomes containing homogenously ubiquitylated $\mathrm{H} 2 \mathrm{~A}$ and $\mathrm{H} 2 \mathrm{~B}$ prepared using semisynthesis. J. Am. Chem. Soc. 134, 19548-19551 (2012).

62. Hansen, J. C. Conformational dynamics of the chromatin fiber in solution: determinants, mechanisms, and functions. Annu. Rev. Biophys. Biomol. Struct. 31, 361-392 (2002).

63. Vasudevan, D., Chua, E. Y. \& Davey, C. A. Crystal structures of nucleosome core particles containing the '601' strong positioning sequence. J. Mol. Biol. 403, 1-10 (2010)

64. Eggeling, C. et al. Data registration and selective single-molecule analysis using multi- parameter fluorescence detection. J. Biotechnol. 86, 163-180 (2001).

65. Al-Soufi, W. et al. Fluorescence correlation spectroscopy, a tool to investigate supramolecular dynamics: inclusion complexes of pyronines with cyclodextrin. J. Am. Chem. Soc. 127, 8775-8784 (2005).

66. Felekyan, S. et al. Full correlation from picoseconds to seconds by timeresolved and time-correlated single photon detection. Rev. Sci. Instrum. 76, 083104 (2005).

67. Kühnemuth, R. \& Seidel, C. A. M. Principles of single molecule multiparameter fluorescence spectroscopy. Single Mol. 2, 251-254 (2001).

\section{Acknowledgements}

We dedicate this paper to the memory of Jörg Langowski, a brilliant pioneer in studying chromatin in silico, in vitro, and in live cells. He had the courage to follow his heart and intuition in life and research. We thank Nathalie Schwarz for expert technical assistance in preparing the nucleosome samples and Mykola Dimura for AV simulations. This work was supported by DFG grants La 500/18-1 to J.L. and Se 1195/15-1 to C.A.M.S.; This research was also supported by the European Research Council through the Advanced Grant 2014 hybridFRET (671208) to C.A.M.S.

\section{Author contributions}

A.G., K.L., and K.T. prepared samples. A.G. and K.L. performed and analyzed ensemble FRET experiments. A.G., K.L., K.T., J.L., S.F., and R.K. performed smFRET experiments. S.F., R.K., and C.A.M.S. analyzed smFRET data. R.K. performed kinetic analysis. A.G., S.F., K.L., and R.K. prepared the figures and discussed them with all authors. All authors participated in the writing of the manuscript. SI was collected by S.F., R.K., and A.G.; J.L., K.T., and C.A.M.S. supervised the project.

\section{Additional information}

Supplementary Information accompanies this paper at https://doi.org/10.1038/s41467018-06758-1.

Competing interests: The authors declare no competing interests.

Reprints and permission information is available online at http://npg.nature.com/ reprintsandpermissions/

Publisher's note: Springer Nature remains neutral with regard to jurisdictional claims in published maps and institutional affiliations.

(c) Open Access This article is licensed under a Creative Commons Attribution 4.0 International License, which permits use, sharing, adaptation, distribution and reproduction in any medium or format, as long as you give appropriate credit to the original author(s) and the source, provide a link to the Creative Commons license, and indicate if changes were made. The images or other third party material in this article are included in the article's Creative Commons license, unless indicated otherwise in a credit line to the material. If material is not included in the article's Creative Commons license and your intended use is not permitted by statutory regulation or exceeds the permitted use, you will need to obtain permission directly from the copyright holder. To view a copy of this license, visit http://creativecommons.org/ licenses/by/4.0/.

(c) The Author(s) 2018 